\title{
Current-induced magnetization caused by crystal chirality in nonmagnetic elemental tellurium
}

\author{
Tetsuya Furukawa $\odot,{ }^{1,2,{ }^{*}}$ Yuta Watanabe, ${ }^{2}$ Naoki Ogasawara, ${ }^{2}$ Kaya Kobayashi $\odot,{ }^{3}$ and Tetsuaki Itou $\odot^{2, \dagger}$ \\ ${ }^{1}$ Institute for Materials Research, Tohoku University, Sendai 980-8577, Japan \\ ${ }^{2}$ Department of Applied Physics, Tokyo University of Science, Tokyo 125-8585, Japan \\ ${ }^{3}$ Research Institute for Interdisciplinary Science, Okayama University, Okayama 700-8530, Japan
}

(Received 20 October 2020; accepted 14 April 2021; published 10 May 2021)

\begin{abstract}
Chiral matter has a structure that lacks inversion, mirror, and rotoreflection symmetry; thus, a given chiral material has either a right- or left-handed structure. In chiral matter, electricity and magnetism can be coupled in an exotic manner beyond the classical electromagnetism (e.g., magneto chiral effect in chiral magnets). In this paper, we give a firm experimental proof of the linear electric-current-induced magnetization effect in bulk nonmagnetic chiral matter elemental trigonal tellurium. We measured a ${ }^{125} \mathrm{Te}$ nuclear magnetic resonance (NMR) spectral shift under a pulsed electric current for trigonal tellurium single crystals. We provide general symmetry considerations to discuss the electrically (electric-field- and electric-current-) induced magnetization and clarify that the NMR shift observed in trigonal tellurium is caused by the linear current-induced magnetization effect, not by a higher-order magnetoelectric effect. We also show that the current-induced NMR shift is reversed by a chirality reversal of the tellurium crystal structure. This result is direct evidence of crystal-chirality-induced spin polarization, which is an inorganic-bulk-crystal analog of the chirality-induced spin selectivity in chiral organic molecules. The present findings also show that nonmagnetic chiral crystals may be applied to spintronics and coil-free devices to generate magnetization beyond the classical electromagnetism.
\end{abstract}

DOI: 10.1103/PhysRevResearch.3.023111

\section{INTRODUCTION}

In condensed matters, various crystal symmetries and low-energy excitations allow an exotic coupling between electricity and magnetism beyond the classical electromagnetism. These cross-correlation phenomena, called magnetoelectric effects [1] (in a broad sense), may have applications in information and energy technology. Among various magnetoelectric effects, the linear electric-field-induced magnetization effect can occur only in a system that lacks both inversion and time-reversal symmetry [2]. Thus, this magnetoelectric effect is forbidden in nonmagnetic materials. (The term "magnetization" is defined in this paper as net macroscopic magnetization, unless there is particular attention. Note that magnetization is, in principle, caused not only by spin polarization but also by orientation of orbital magnetic moment.) In contrast, an electric current can induce magnetization in a noncentrosymmetric material, even if the material has time-reversal symmetry. This current-induced magnetization effect has so far been studied in surface/interface Rashba systems [3-12] as the Edelstein effect [13]. In a microscopic viewpoint, an electric current causes an imbalance between opposite spin populations in spin-split bands caused by the

\footnotetext{
*tf@imr.tohoku.ac.jp

†tetsuaki.itou@rs.tus.ac.jp
}

Published by the American Physical Society under the terms of the Creative Commons Attribution 4.0 International license. Further distribution of this work must maintain attribution to the author(s) and the published article's title, journal citation, and DOI. breaking of inversion symmetry and the spin-orbit interaction (SOI). A promising direction of the current-induced magnetization effect is to expand the phenomenon into bulk materials. The availability of various types of crystal symmetry in bulk materials allows us to arbitrarily design a relation between the directions of an electric current and induced magnetization.

A sign of the bulk current-induced magnetization has been observed as current-induced modulation of optical activity in elemental trigonal tellurium $[14,15]$. We recently reported the possibility of the bulk linear current-induced magnetization in trigonal tellurium by observing a hyperfine shift, which is proportional to the local electronic magnetization, of the nuclear magnetic resonance (NMR) spectrum induced by an electric current [16]. The induced magnetization can be parallel to an applied electric current in tellurium because of its chiral nature, which contrasts sharply with the currentinduced magnetization perpendicular to an applied current in Rashba systems. Thus, the possible current-induced magnetization effect in tellurium may provide coil-free devices to generate magnetization and a magnetic field. Moreover, the possible current-induced spin polarization in chiral tellurium may be a bulk-crystal counterpart of the chirality-induced spin selectivity studied in chiral organic molecules [17]. However, the analysis and the argument of our previous work were not perfect, as will be explained later. An incontrovertible proof of this phenomenon is desired.

Here we add further confirmation to our claim that the linear current-induced magnetization effect occurs in chiral matter tellurium [16] by providing symmetry considerations to discuss the general magnetoelectric effect and by analyzing the observed electrically (electric-field- and electric-current-) 
(a)
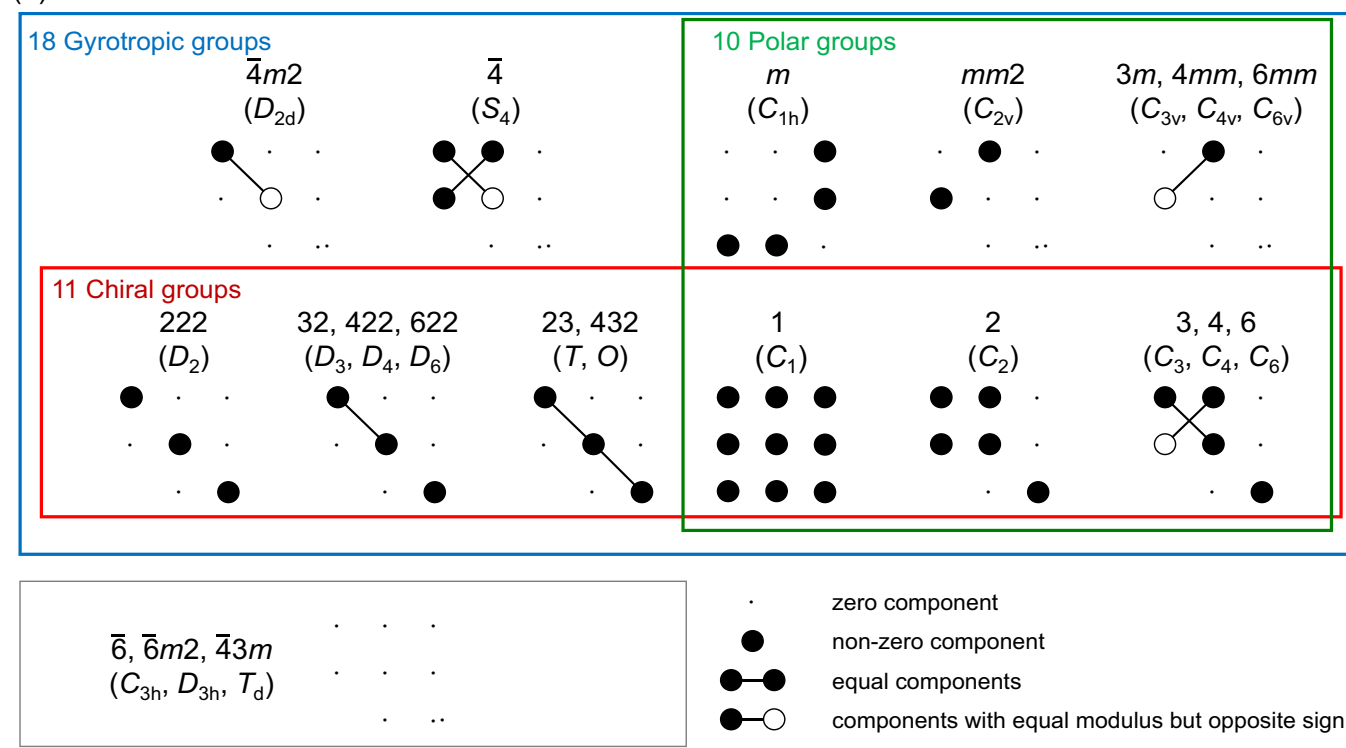

(b)

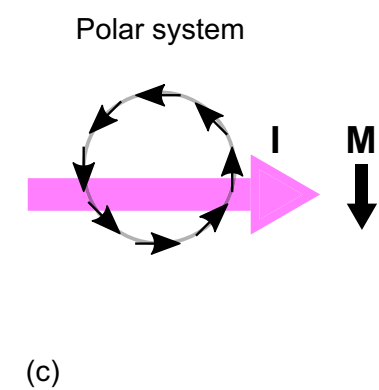

(c)

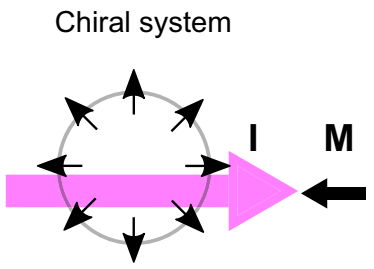

FIG. 1. Relation between the linear current-induced magnetization and the crystal point groups. (a) Possible second-rank axial tensor forms of the linear current-induced magnetization effect for 21 noncentrosymmetric crystal point groups. Only 18 gyrotropic groups can have nonzero components of the tensor. Three of the noncentrosymmetric groups are nongyrotropic. The tensor forms for the point groups of the monoclinic systems, $2\left(C_{2}\right)$ and $m\left(C_{1 \mathrm{~h}}\right)$, are represented in the first setting, where the twofold rotation axis (the mirror plane) is parallel (perpendicular) to the $z$ axis. (b) Circular angular-momentum texture in the $\mathbf{k}$ space in a polar system. The current-induced magnetization $\mathbf{M}$ tends to be perpendicular to an applied electric current $\mathbf{I}$. (c) Radial angular-momentum texture in a chiral system. $\mathbf{M}$ tends to be parallel to $\mathbf{I}$.

induced NMR spectral shifts from the viewpoint of the symmetry considerations. We address two issues. First, we prove that the electrically induced NMR shift is attributable to the linear current-induced magnetization effect, not to higherorder magnetoelectric effects. Second, we present that the chirality reversal of the crystal structure causes the polarity reversal of the current-induced magnetization using a rightand a left-handed single crystal. These two results give a conclusive understanding of the magnetoelectric effect in trigonal tellurium.

\section{SYMMETRY CONSIDERATIONS}

The bulk linear current-induced magnetization is written as $\mathbf{M}=\beta \mathbf{I}$. The second-rank current-induced magnetization tensor $\beta$ must be axial because the tensor connects magnetization $\mathbf{M}$ (an axial vector) and electric current I (a polar vector). Axial tensors acquire a minus sign through improper rotation; hence, the matrix elements of $\beta$ are more strongly constrained by improper rotation symmetry (e.g., inversion, mirror, and rotatory reflection symmetry) than by proper rotation symmetry, sometimes being zero. For example, inversion symmetry forces all elements of $\beta$ to be zero. Figure 1(a) shows the possible tensor forms of $\beta$ for 21 noncentrosymmetric crystal point groups. Notably, all elements of $\beta$ are zero for $\overline{6}\left(C_{3 \mathrm{~h}}\right), \overline{6} m 2\left(D_{3 \mathrm{~h}}\right)$, and $\overline{4} 3 m\left(T_{\mathrm{d}}\right)$, even though they do not possess inversion symmetry. Thus, the bulk linear currentinduced magnetization effect can occur only in systems belonging to the remaining 18 noncentrosymmetric crystal point groups, called the gyrotropic groups. In the gyrotropic groups, the two subclasses of polar and chiral groups are important. Polar systems, such as the Rashba systems, have a circular angular-momentum texture in the wave number $\mathbf{k}$ space and antisymmetric (off-diagonal) components in $\beta$; thus, an electric current and an induced magnetization tend to be orthogonal independently of the current direction [Fig. 1(b)]. Chiral systems (e.g., trigonal tellurium), which have no improper rotation symmetry, have a radial angularmomentum texture and isotropic diagonal components in $\beta$; thus, an electric current and an induced magnetization tend to be parallel [Fig. 1(c)].

\section{CRYSTAL AND ELECTRONIC STRUCTURES OF TRIGONAL TELLURIUM}

Trigonal tellurium is a semiconductor with a narrow bandgap of $E_{\mathrm{g}}=0.32 \mathrm{eV}$, with only the $p$-type being available. Trigonal tellurium has a crystal structure belonging to either space group $P 3_{1} 21\left(D_{3}^{4}\right)$ or $P 3_{2} 21\left(D_{3}^{6}\right)$ [Figs. 2(a) and 2(b)]. Tellurium atoms form dominant covalent bonds in a helix structure with a threefold screw symmetry. The helices along the crystal $c$ axis further form a hexagonal arrangement with van der Waals interactions, thereby yielding a chiral crystal structure. Note that a crystal with right-handed helices of the covalent bonds belongs to the space group with a right-handed symmetry element $P 3_{1} 21\left(D_{3}^{4}\right)$, and a crystal with left-handed helices belongs to $P 3_{2} 21\left(D_{3}^{6}\right)$ [18]. In this paper, we define the chirality of the crystal structure by referring to that of the covalent-bond helices. We use the phrases "right-handed tellurium" for $P 3_{1} 21\left(D_{3}^{4}\right)$ tellurium and "left-handed tellurium" for $P 3_{2} 21\left(D_{3}^{6}\right)$ tellurium (see Appendix B). The chirality of the covalent-bond helices determines the polarities of many physical properties, such as the angular-momentum texture in the k space [19-21], natural 
(a)

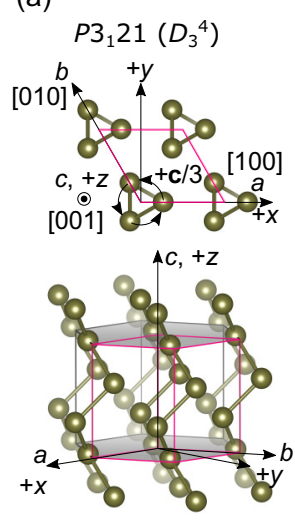

(b)

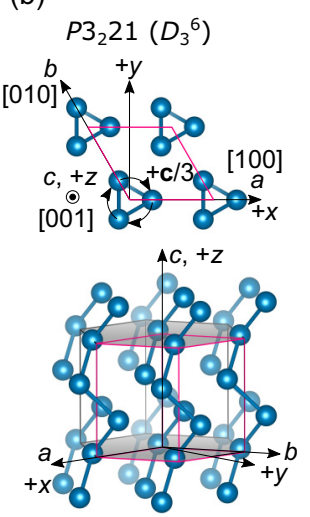

(c)

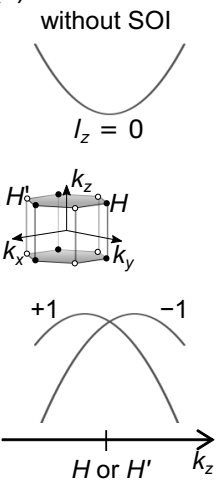

(d)
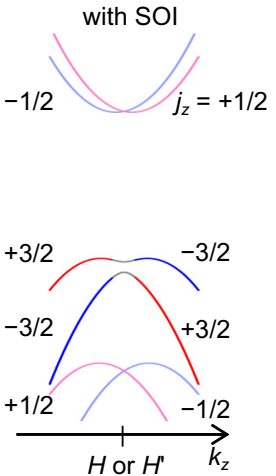

(e)

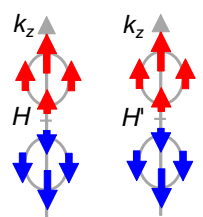

(g)

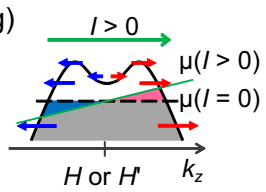

$\left\langle s_{z}\right\rangle>0\left\langle M_{\mathrm{s}, \mathrm{z}}\right\rangle<0$ (f)

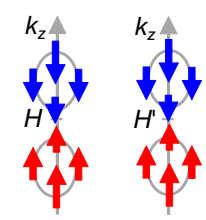

(h)

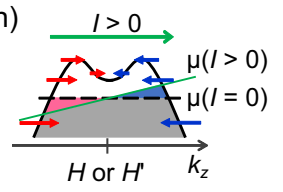

$\left\langle s_{z}\right\rangle<0\left\langle M_{s, z}\right\rangle>0$

FIG. 2. Crystal and electronic structure of trigonal tellurium. (a), (b) Crystal structures of tellurium for $P 3_{1} 21\left(D_{3}^{4}\right)\left(\right.$ a) and $P 3_{2} 21\left(D_{3}^{6}\right)$ (b) with right-handed covalent-bond helices and left-handed helices, respectively. The pink parallelogram indicates a unit cell. A right-handed crystal has the screw operation of $\left\{\rho_{3} \mid \mathbf{c} / 3\right\}$ as a symmetry operation, while a left-handed crystal has the operation of $\left\{\rho_{3} \mid 2 \mathbf{c} / 3\right\}$, where $\rho_{3}$ is a $120^{\circ}$ counterclockwise rotation along the $c$ axis, and $\mathbf{c}$ is the primitive vector along the $c$ direction. (c), (d) Schematic band structures along $k_{z}$ (and $k_{x}=k_{y}=0$ ) around the $H$ and $H^{\prime}$ points with (d) and without (c) the spin-orbit interaction (SOI). The signs of the (interatomic) orbital angular momentum $\left(l_{z}\right)$ and the total angular momentum $\left(j_{z}\right)$ for each wave number $\mathbf{k}$ and for each band are reversed when the crystal structure chirality is reversed. The bands in the panel (c) are totally spin-degenerate. The hexagonal prism in (c) shows a schematic image of the Brillouin zone of trigonal tellurium. (e), (f) Schematic outward (e) and inward (f) angular-momentum texture around the $H$ and $H^{\prime}$ points when chemical potential crosses the camel-back structure of the highest valence band. $(\mathrm{g})$, (h) Relation among the polarities of the net spin-angular momentum $\left\langle s_{z}\right\rangle$ and of the net spin magnetization $\left\langle M_{s, z}\right\rangle$ and an imbalance between the opposite spin populations for the highest valence band under a positive electric current $\left(\mathbf{I} \|+\hat{\mathbf{k}}_{z}\right)$ for outward $(\mathrm{g})$ and inward $(\mathrm{h})$ spin-angular-momentum textures.

optical rotatory power [22-25], second-harmonic generation [26], piezoelectricity [27,28], shapes of etch pits [29], resonant diffraction with circularly polarized $\mathrm{x}$ rays [30,31], polarized neutron scattering [32], and NMR chemical shift [33] (see Appendix B). Trigonal tellurium has the bottoms of conduction bands and the tops of valence bands around the $H$ and $H^{\prime}$ points $[19,34,35]$. These points are at the corners of the hexagonal prism first Brillouin zone and are related to each other by a time-reversal operation; they are not time-reversal invariant momentums. Thus, the time-reversal symmetry of trigonal tellurium does not provide Kramers degeneracy at the $H$ and $H^{\prime}$ points. Around these points, if spin-orbit interactions are absent, the conduction bands would form a doubly spin-degenerate parabolic band, whereas the valence bands would form spin-degenerate, but (interatomic) orbital angularmomentum split bands [36] with quadruple degeneracy at the $H$ and $H^{\prime}$ points owing to threefold screw and twofold rotation symmetry [Fig. 2(c)]. The spin-orbit interactions lift the spin degeneracy, thereby causing the two spin-split conduction bands crossing at the $H$ and $H^{\prime}$ points, the highest and second highest nondegenerate valence bands, and the two splitting deeper valence bands with band crossing [Fig. 2(d)]. This band splitting provides total-angular-momentum textures in the $\mathbf{k}$ space. The two deeper valence bands primarily consist of states with total angular momentum $j_{z}=+1 / 2$ or $-1 / 2$ and have a hedgehog angular-momentum texture around the $H$ and $H^{\prime}$ points (i.e., the spin-split conduction bands also have a similar angular-momentum texture [19]). Notably, the crystal threefold screw and the twofold rotation symmetries forbid hybridization between the Bloch states with $j_{z}=+1 / 2$ and $-1 / 2$ at the $H$ and $H^{\prime}$ points, that is, they protect the band crossing. This crossing point is nothing but a Weyl node; thus, the possibility of a topological Weyl semimetal phase is argued for trigonal tellurium [19,21,37,38]. In contrast, the highest and second highest valence bands primarily consist of the hybridized states of $j_{z}= \pm 3 / 2$, and the wave functions $\left|j_{\mathrm{z}}=+3 / 2\right\rangle \pm\left|j_{\mathrm{z}}=-3 / 2\right\rangle$ are realized at the $H$ and $H^{\prime}$ points. Such spin-nematic-type hybridization opens a gap between the two valence bands and causes a radial, but almost $k_{z}$-directional spin texture [Figs. 2(e) and 2(f)]. This $c$-axis anisotropy in $p$-type tellurium with a low carrier density causes that only the $c$-axis part of an electric current induces magnetization, which also has only the $c$-axis component. The $\mathbf{k}$ space angular-momentum textures for a rightand a left-handed crystal have opposite polarity, thanks to the axial vector nature of the angular momentum. (Ab initio calculations $[20,21]$ directly show that the right- $\left[P 3_{1} 21\left(D_{3}^{4}\right)\right]$ and left-handed $\left[P 3_{2} 21\left(D_{3}^{6}\right)\right]$ crystals have outward and inward angular-momentum textures, respectively [Figs. 2(e) and 2(f)].) Thus, an electric current should cause a linear currentinduced magnetization with polarity depending on the crystal structure chirality [Figs. 2(g) and 2(h)].

\section{MEASURING METHOD}

We measured the ${ }^{125}$ Te NMR spectra for two single crystals (samples 1 and 2) at $100 \mathrm{~K}$ under an applied pulsed electric current. Samples 1 and 2 were synthesized with the physical vapor transport technique and the repeated Bridgman method, respectively (The detail of the synthesis is explained in Appendix A). Both methods are not chirality-selective-growth methods; thus, to obtain both right- and left-handed single crystals, we picked up a number of crystals and determined the crystal structure chirality for each sample by 
(a)

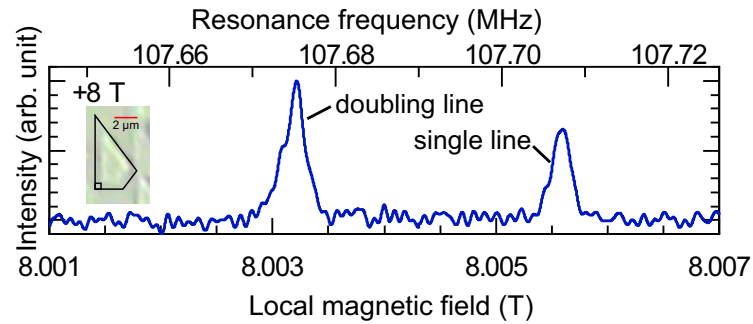

(c)

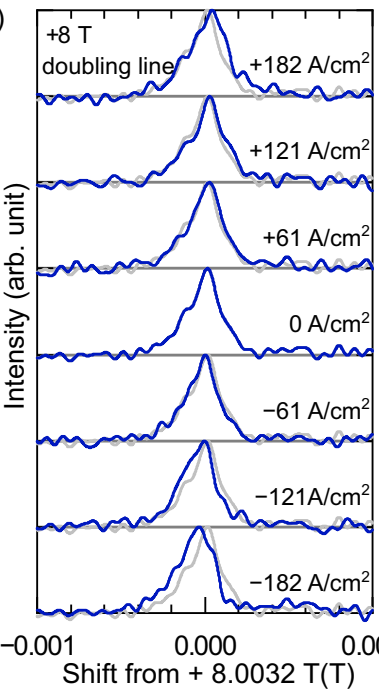

(d)

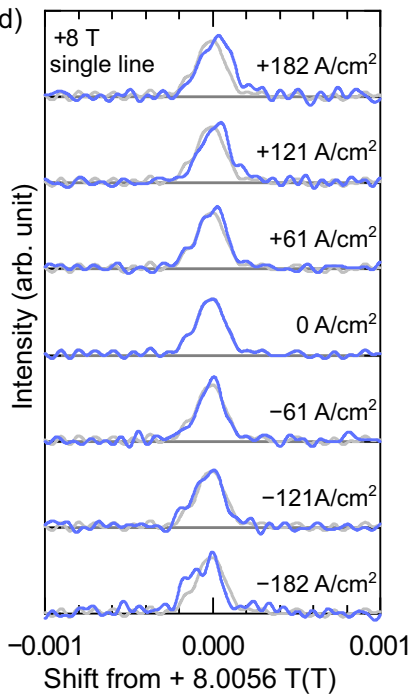

(b)

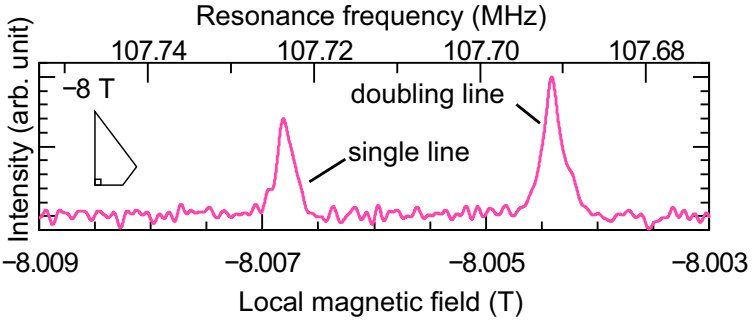

(e)

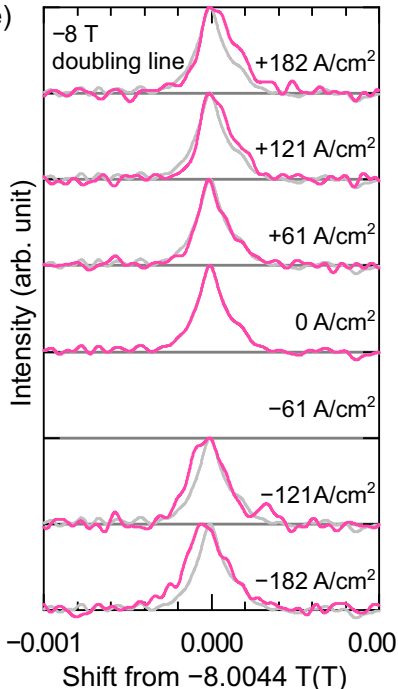

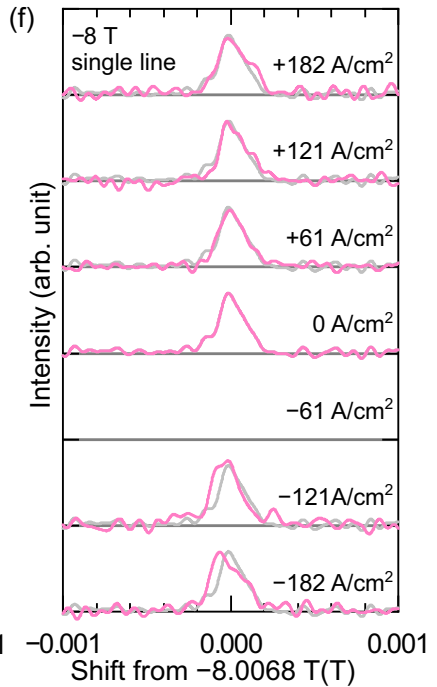

FIG. 3. Magnetic-field polarity dependence of the electrically induced NMR shift. (a), (b) Single-crystal ${ }^{125} \mathrm{Te}-\mathrm{NMR}$ spectra for sample 1 for (a) a positive and (b) a negative magnetic field in the absence of a pulsed electric current at $100 \mathrm{~K}$. A magnetic field was applied approximately parallel to the $c$ axis, but slightly tilted to the $y$ axis. The spectra are plotted as a function of the effective magnetic field felt by the ${ }^{125}$ Te nuclei (see Appendix A). The observed NMR spectra consist of two lines, that is, a single line and a doubling line, which are respectively associated with one and two of the three tellurium atoms in a unit cell. The inset in (a) is a photo image of an etch pit on the crystal, whose type appears on the left-handed $\left[P 3_{2} 21\left(D_{3}^{6}\right)\right]$ crystal [29]. (c)-(f) Doubling line (c) and single line (d) in the ${ }^{125}$ Te-NMR spectrum for a positive magnetic field and doubling line (e) and single line (f) for a negative magnetic field for different electric current densities. For comparison, the gray lines in each row show the spectrum in the absence of a pulsed electric current. The absence of data for $-61 \mathrm{Acm}^{-2}$ under a negative field is caused by the irreversible damage of an electric contact of the specimen during the measurement for this condition.

observing the shapes of etch pits on the surfaces of each sample. According to the etch pit study [29], samples 1 and 2 are left- $\left[\left(P 3_{2} 21\left(D_{3}^{6}\right)\right]\right.$ and right-handed $\left[P 3_{1} 21\left(D_{3}^{4}\right)\right]$ tellurium, respectively [Figs. 3(a) and 5(a)]. We applied a static magnetic field of $8.012 \mathrm{~T}$ for sample 1 (the left-handed crystal) and $8.327 \mathrm{~T}$ for sample 2 (the right-handed crystal) almost parallel to the $c$ axis. A pulsed current synchronized with the NMR measurement was also applied almost parallel to the $c$ axis through two electric terminals (see Appendix A). To avoid movement of the sample by the pulsed electric current, sample 1 with a coil for the NMR measurements was coated with epoxy (Stycast 1266) and fixed on a glass plate, and sample 2 with a coil was soaked into a pressure medium of Daphne 7373 and placed in a $\mathrm{BeCu}$ pressure cell, in which a low pressure of $0.5 \mathrm{GPa}$ was applied at room temperature. The temperature dependence of the twoterminal resistance showed that the present crystals had an impurity hole density less than $1 \times 10^{16} \mathrm{~cm}^{-3}$ and that the systems at $100 \mathrm{~K}$ were in the extrinsic region with a negligible density of the thermally excited carriers across the band gap. For an arbitrary magnetic field direction, the NMR spectrum of trigonal tellurium should have three lines with the same intensity that correspond to the three atoms in the unit cell. For the present samples, we verified that such a three-line spectrum appeared when the magnetic field direction was rotated away from the $c$ axis, confirming that the samples were single crystals. In the following results, an applied magnetic field was almost parallel to, but slightly tilted from the $c$ axis. The tilting direction was controlled to be precisely aligned to the $y$ axis. Thus, the observed NMR spectra consisted of two lines, a single line and a doubling line, which are respectively associated with one and two of the three tellurium atoms in a unit cell [Figs. 3(a) and 3(b) and 5(a)]. We estimated the angular differences between the magnetic field directions and the $c$ axis to be approximately $7^{\circ}$ for sample 1 and $9^{\circ}$ for sample 2 by comparing the two-line spectrum with the shift parameters [33].

\section{RESULTS}

\section{A. Magnetic-field polarity dependence of current-induced NMR shift}

We observed the electrically induced ${ }^{125} \mathrm{Te}$ NMR spectral shift in Figs. 3(c)-3(f) in more detail than in our previous report [16]. Below, we show that the electrically induced NMR shift is caused by the linear 
current-induced magnetization effect, and not by a higherorder magnetoelectric effect. In theoretical viewpoints, the electric-field- and electric-current-induced magnetization can be clearly distinguished. The former is an equilibrium phenomenon without energy dissipation and requires systems that lack both inversion $(P)$ and time-reversal $(T)$ symmetry. In contrast, the latter is a nonequilibrium phenomenon that requires systems lacking both $P$ and $P T$ symmetry. These relations indicate the duality of the two phenomena: the electric-field-induced magnetization needs an odd-parity component of magnetic ordering in real space, whereas the electric-current-induced magnetization needs an odd-parity component of the magnetization textures (angular-momentum textures) in the $\mathbf{k}$ space. However, in real experiments, the two effects cannot be immediately distinguished because an electric current inevitably needs an electric field. Furthermore, the NMR measurement requires an external magnetic field, which explicitly breaks the time-reversal symmetry of materials and may cause higher-order magnetoelectric effects. Thus, to prove firmly the linear current-induced magnetization effect, it is necessary to rule out the scenarios that the electrically induced magnetization observed originates from the electric-field-induced magnetization and/or higher-order magnetoelectric effects. (This was not done in our previous report [16].) From this viewpoint, we first consider the symmetry of the magnetism induced by an electric current $\mathbf{I}$, an electric field $\mathbf{E}$, and a magnetic field $\mathbf{H}$. Phenomenologically, magnetization can be expanded up to the second order of $\mathbf{I}, \mathbf{E}$, and $\mathbf{H}$ as follows [39]:

$$
\begin{aligned}
M_{i}= & \chi_{i j} H_{j}+\alpha_{i j} E_{j}+\beta_{i j} I_{j}+\Gamma_{i, j k}^{\mathrm{IE}} I_{j} E_{k}+\Gamma_{i, j k}^{\mathrm{EH}} E_{j} H_{k} \\
& +\Gamma_{i, j k}^{\mathrm{IH}} I_{j} H_{k}+\Gamma_{i, j k}^{\mathrm{II}} I_{j} I_{k}+\Gamma_{i, j k}^{\mathrm{EE}} E_{j} E_{k}+\Gamma_{i, j k}^{\mathrm{HH}} H_{j} H_{k},
\end{aligned}
$$

where, $i, j$, and $k(=x, y, z)$ are the indices of the matrix elements; $\chi, \alpha$, and $\beta$ are the second-rank response tensors for magnetic susceptibility, linear electric-field-induced magnetization, and linear current-induced magnetization, respectively; and $\Gamma^{\mathrm{IE}}, \Gamma^{\mathrm{EH}}, \Gamma^{\mathrm{IH}}, \Gamma^{\mathrm{II}}, \Gamma^{\mathrm{EE}}$, and $\Gamma^{\mathrm{HH}}$ are the third-rank response tensors for each bilinear effect.

To know which terms can survive under the $P$ and $T$ transformations, we considered the $P$ and $T$ transformations of $\mathbf{M}$, $\mathbf{I}, \mathbf{E}$, and $\mathbf{H}$. I is inversion-odd ( $P$-odd) and time-reversal odd ( $T$-odd); $\mathbf{E}$ is $P$-odd and $T$-even; and $\mathbf{M}$ and $\mathbf{H}$ are $P$-even and $T$-odd. In accordance with these transformations, some response tensors must be zero when a system in a null field has $P$ and/or $T$ symmetry. For example, the linear electricfield-induced magnetization tensor $\alpha$ must be zero when a given system has a time-reversal symmetry because input $\mathbf{E}$ and output $\mathbf{M}$ acquire opposite signs through a time-reversal transformation.

For trigonal tellurium, $\alpha$ and $\Gamma^{\mathrm{IH}}$ must be zero, and $\beta$ and $\Gamma^{\mathrm{EH}}$ can be nonzero because tellurium is a nonmagnetic chiral (or, $T$-symmetric and $P$-broken) semiconductor. We can also neglect the effect of $\chi_{i j} H_{j}, \Gamma_{i, j k}^{\mathrm{IE}} I_{j} E_{k}, \Gamma_{i, j k}^{\mathrm{II}} I_{j} I_{k}, \Gamma_{i, j k}^{\mathrm{EE}} E_{j} E_{k}$, and $\Gamma_{i, j k}^{\mathrm{HH}} H_{j} H_{k}$ because the observed electrically induced NMR shift shows a linear dependence on an electric input, $\mathbf{I}$ or $\mathbf{E}$ (The linearity was reported in our previous work [16] and confirmed in Figs. 4 and 6 here.) Thus, the possible origins of the electrically induced NMR shift are the linear current-induced magnetization term $\beta_{i j} I_{j}$ and/or the bilinear magnetoelec-

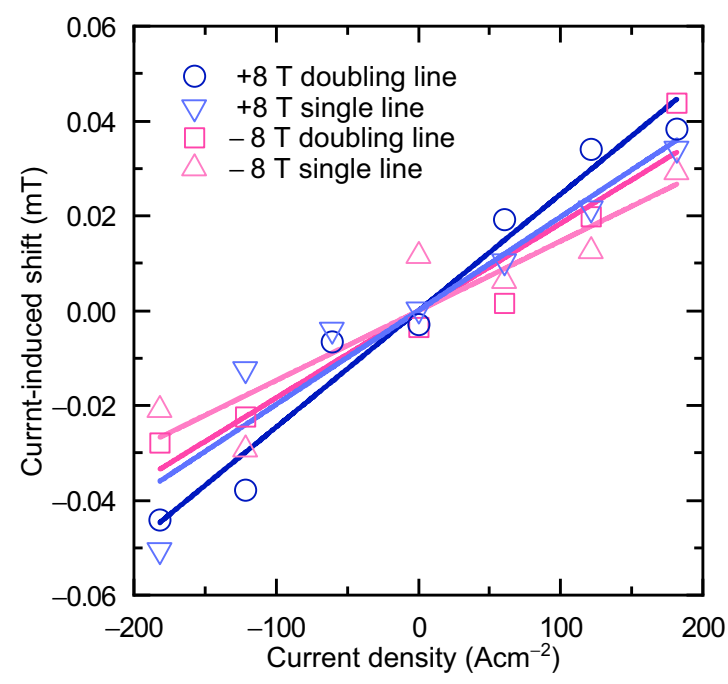

FIG. 4. Current density dependence of the current-induced shifts for sample 1. The current-induced shift is defined as the difference between the spectral first moment of each line and the shift origin defined such that the intersection of the fitting lines should be zero. The proportionality coefficients obtained by least-squares fitting are $+2.5( \pm 0.2) \times 10^{-4} \mathrm{mTA}^{-1} \mathrm{~cm}^{2}$ for the doubling line $(+8 \mathrm{~T}),+2.0$ $( \pm 0.3) \times 10^{-4} \mathrm{mTA}^{-1} \mathrm{~cm}^{2}$ for the single line $(+8 \mathrm{~T}),+2.0( \pm 0.3)$ $\times 10^{-4} \mathrm{mTA}^{-1} \mathrm{~cm}^{2}$ for the doubling line $(-8 \mathrm{~T})$, and $+1.8( \pm 0.3)$ $\times 10^{-4} \mathrm{mTA}^{-1} \mathrm{~cm}^{2}$ for the single line $(-8 \mathrm{~T})$.

tric term $\Gamma_{i, j k}^{\mathrm{EH}} E_{j} H_{k}$. (Specifically, the bilinear magnetoelectric effect primarily originates from a modulation of the magnetic susceptibility induced by the piezoelectric effect.) We measured the magnetic-field polarity dependence of the electrically induced shift to clarify which effect dominates the electrically induced NMR shift observed in trigonal tellurium. The linear current-induced magnetization $\beta_{i j} I_{j}$ is independent of the polarity of a magnetic field; thus, the polarity reversal of a magnetic field does not change the polarity of the NMR shift induced by this effect. In contrast, the bilinear magnetoelectric $\Gamma_{i, j k}^{\mathrm{EH}} E_{j} H_{k}$ term depends on the magnetic-field polarity; thus, the polarity reversal of the magnetic field reverses the polarity of the NMR shift induced by the bilinear magnetoelectric effect.

Figures 3(c)-3(f) show the current density dependence of the NMR spectra under the positive and negative magnetic fields of $8.012 \mathrm{~T}$ for sample 1 , which has a left-handed $\left[P 3_{2} 21\left(D_{3}^{6}\right)\right]$ crystal structure [Fig. 3(a)]. The polarity reversal of the magnetic field preserves the polarity of the observed electrically induced NMR shift. This experimental result demonstrates that the electrically induced NMR shift observed in trigonal tellurium is caused by the linear current-induced magnetization effect. Figure 4 shows the current density dependence of the electrically induced NMR shift. The slope coefficients for the doubling line are $+2.5 \pm 0.2 \times 10^{-4} \mathrm{mTA}^{-1} \mathrm{~cm}^{2}$ for a positive field and $+2.0 \pm 0.3 \times 10^{-4} \mathrm{mTA}^{-1} \mathrm{~cm}^{2}$ for a negative field, showing a good agreement between the two values within the margin error. The slope coefficients for the single line are $+2.0 \pm$ $0.3 \times 10^{-4} \mathrm{mTA}^{-1} \mathrm{~cm}^{2}$ for a positive field and $+1.8 \pm 0.3 \times$ $10^{-4} \mathrm{mTA}^{-1} \mathrm{~cm}^{2}$ for a negative field, which also show a good agreement. The agreement between the coefficients for the 
positive and negative magnetic fields indicates that the bilinear magnetoelectric effect makes a minor or negligible contribution to the electrically induced NMR shift compared to the linear current-induced magnetization effect. The slight difference between the slope coefficients of the doubled line and the single line is also observed, although it is comparable with the fitting error bars. This may indicate that the off-diagonal hyperfine coupling coefficient causes different hyperfine field components parallel to the external magnetic field slightly tilting from the $c$ axis, for the doubling-line and single-line tellurium atoms. Revisiting the symmetry consideration, the $\Gamma_{i, z z}^{\mathrm{EH}}$ coefficients must be zero for the $32\left(D_{3}\right)$ point group symmetry of tellurium. In other words, all directional components of the bilinear EH-induced magnetization must be zero when external electric and magnetic fields are applied exactly parallel to the $c$ axis of tellurium. Although the present experimental setup had a slight misalignment of the magnetic and electric fields from the $c$ axis, this symmetry constraint guaranteed the weakness of the bilinear magnetoelectric effects for the present directions of the fields, even if the bilinear effect for the arbitrary field directions of an external fields would be detectable.

\section{B. Crystal structure chirality dependence of current-induced NMR shift}

We next show that the polarity of the current-induced magnetization in tellurium depends on the crystal structure chirality. Figures 5(b) and 5(c) show the ${ }^{125}$ Te NMR spectra under a positive magnetic field of $8.327 \mathrm{~T}$ for sample 2 , which has a right-handed $\left[P 3_{1} 21\left(D_{3}^{4}\right)\right]$ crystal structure [Fig. 5(a)]. Similar to the results for sample 1 [Figs. 3(c)3(f)], an electric-current induces local-field shifts, and the shifts linearly depend on the electrical current density with coefficients of $-5.1 \pm 0.4 \times 10^{-4} \mathrm{mTA}^{-1} \mathrm{~cm}^{2}$ for the doubling line and $-4.2 \pm 0.5 \times 10^{-4} \mathrm{mTA}^{-1} \mathrm{~cm}^{2}$ for the single line (Fig. 6). Notably, the polarity of the shifts induced by a positive electric current was negative for sample 2 (right-handed tellurium) in contrast to the positive shifts for sample 1 (left-handed tellurium). The negative shifts indicate a negative spin magnetization because of the positive hyperfine coupling coefficient [40] between the $c$-axis electronic spin magnetization and the $c$-axis hyperfine field. Therefore, the polarity of the current-induced spin magnetization is positive for the left-handed tellurium and negative for the right-handed tellurium when a positive current is applied. (We will discuss the possible current-induced orbital magnetization later.) Consequently, the present result shows that a chirality reversal of the crystal structure causes a reversal of the current-induced magnetization. The absolute values of the current-induced magnetization coefficients for the rightand left-handed samples differed by a factor of approximately two. This discrepancy may originate from a non-negligible amount of a surface contribution to the total electric current depending on the surface condition for each sample. If a surface current exists, a bulk current could be overestimated, and the coefficients could be underestimated. In fact, a metallic surface state in the $\{10 \overline{1} 0\}$ plane was observed [41-43]. Note that even if a non-negligible amount of a surface current was present, the Oersted field generated by the surface current (a)

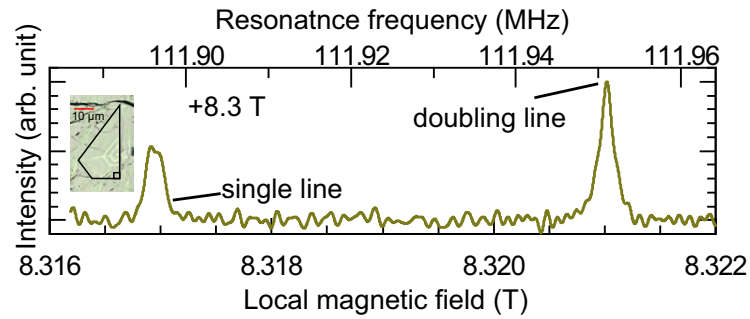

(b)

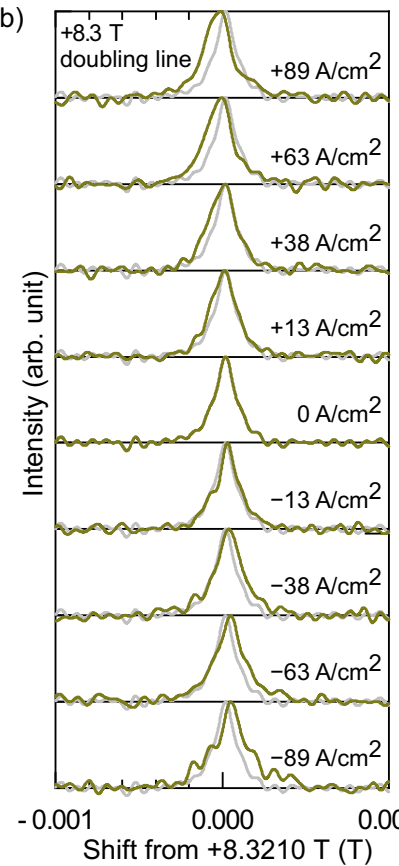

(c)

$+8.3 T$
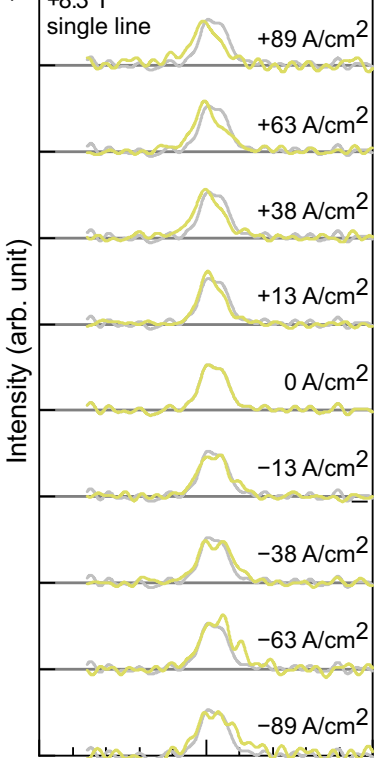

$\begin{array}{lll}-0.001 & 0.000 & 0.001\end{array}$

Shift from $+8.3169 \mathrm{~T}(\mathrm{~T})$
FIG. 5. Inversion of the current-induced shift by a reversal of the crystal structure chirality. (a) Single-crystal ${ }^{125} \mathrm{Te}-\mathrm{NMR}$ spectra for sample 2 in the absence of a pulsed electric current at $100 \mathrm{~K}$. A magnetic field was applied approximately parallel to the $c$ axis, but slightly tilted to the $y$ axis. The spectra are plotted as a function of the effective magnetic field felt by the ${ }^{125}$ Te nuclei (see Appendix A). The observed NMR spectra consist of a single line and a doubling line similar to the spectra for sample 1 . The inset indicates the photo image of an etch pit on the crystal, whose type appears on the right-handed $\left[P 3_{1} 21\left(D_{3}^{4}\right)\right]$ crystal [29]. (b), (c) Doubling (b) and single (c) lines in the ${ }^{125} \mathrm{Te}-\mathrm{NMR}$ spectrum for different electric current densities. For comparison, the gray lines in each row show the spectrum in the absence of a pulsed electric current.

would cause only a broadening (not shift) of the NMR spectra (see Appendix D); thus, the observed current-induced shift originates from the magnetization of the bulk part of the samples.

\section{DISCUSSION}

\section{A. Relation among the current-induced NMR shift, $k$ space spin texture, and crystal structure chirality}

We discuss the relation among the polarity of the currentinduced NMR shift, spin texture of the highest valence bands, and crystal structure chirality. As discussed in our previous report [16], an electric current induces magnetization parallel (antiparallel) to an electric current in tellurium with an inward 


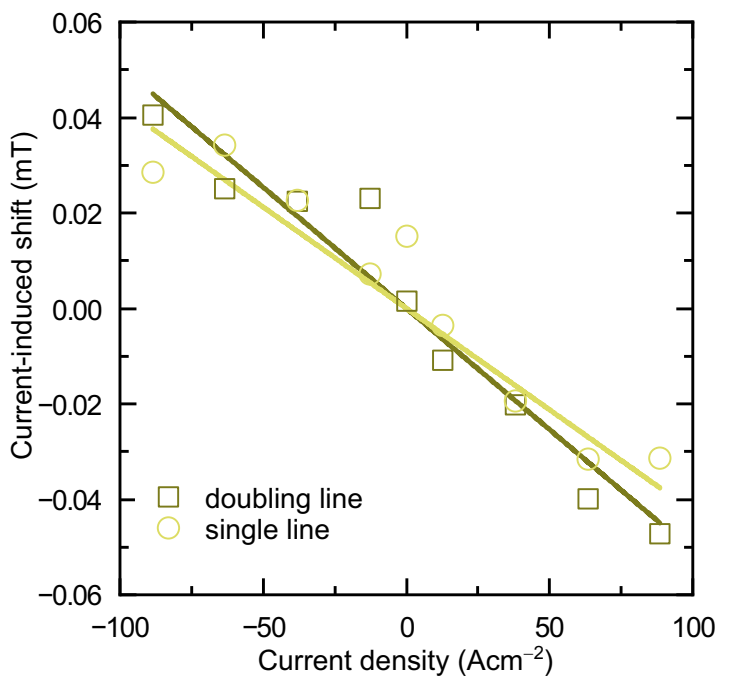

FIG. 6. Current density dependence of the current-induced shifts for sample 2. The current-induced shift is defined as the difference between the spectral first moment of each line and the shift origin defined such that the intersection of the fitting lines should be zero. The proportionality coefficients obtained by least-squares fitting are $-5.1( \pm 0.4) \times 10^{-4} \mathrm{mTA}^{-1} \mathrm{~cm}^{2}$ for the doubling line and -4.2 $( \pm 0.5) \times 10^{-4} \mathrm{mTA}^{-1} \mathrm{~cm}^{2}$ for the single line.

(outward) spin-angular-momentum texture, which provides an outward (inward) spin-magnetization texture around the $H$ and $H^{\prime}$ points. Thus, the present chirality-dependent result shows that a left-handed (right-handed) crystal has an inward (outward) spin-angular-momentum texture, which is consistent with the recent $a b$ initio calculations and the spin- and angle-resolved photoemission spectroscopy measurements [20,21]. We should point out herein that our previous report [16] has two points that need to be corrected. First, we wrote in the previous report that a right-handed crystal should have an inward spin-angular momentum texture, but it actually has an outward texture. When we reported the previous work [16], we were not aware that there are contradictions in earlier literatures about the relations between the crystal chirality and the physical properties. This is the reason for which we deduced the incorrect relation between the crystal chirality and the spin texture in our previous work. We briefly review and discuss the earlier literature in Appendix B. Second, we wrote in the report that the crystal used was right-handed; however, it was left-handed (see Appendix C). In the previous study, we crashed a tellurium ingot into pieces, which was grown by the repeated Bridgman method, and we used one of the pieces for the NMR measurement. At that time, we thought that the sample used for the NMR measurement was right-handed because other pieces of the ingot show the etch pits of the right-handed crystal chirality. However, we recently observed that there are pieces from the same ingot showing the etch pits of the left-handed crystal chirality; thus, we conclude anew that the ingot has multichirality domains and the sample previously used was left-handed. Unfortunately, the etch pits of the crystal used for the previous NMR measurement cannot be investigated because the crystal was destroyed during attempt to remove it from epoxy in which the crystal was embedded. Note that the current-induced positive NMR shift under a positive current in the previous study can be explained consistently by the two facts that the sample is left-handed and that the left-handed tellurium has the inward spin angular-momentum texture.

\section{B. Current-induced orbital magnetization}

The highest valence bands of tellurium have an orbital angular momentum that originates not from an intra-atomic orbital angular momentum of a $p$-wave atomic state, but from an inter-atomic orbital angular momentum of the Bloch state. Thus, an electric current can induce not only net spin magnetization, but also net orbital magnetization, which is called the orbital Edelstein effect [44,45]. In fact, theoretical studies predicted current-induced intrinsic [20,46] and extrinsic [46] orbital magnetization effect in trigonal tellurium. However, we infer here that the observed current-induced NMR shift is not related to the orbital degrees of freedom of electrons. In the present study, the possible origins of currentinduced local magnetic fields at nucleus due to the orbital degrees of freedom of electrons are the following three: (1) the current-induced (or electric-field induced) modulation of a chemical-shift field, (2) the trivial Oersted field induced by a macroscopic electric current (which is the spatial average of a microscopic solenoidal electric current flowing through tellurium helices), and (3) the nontrivial magnetic field induced by the circular component of a microscopic solenoidal electric current. The first, the current-induced (or the electric-field induced) modulation of a chemical-shift field, can be caused by the two processes that an electric input (an electric current or an electric field) causes modulation of chemical bonds and an external magnetic field restores the orbital angular momentum of electrons feeling the potential of the modulated chemical bonds. This effect is exactly a bilinear effect of the EH or IH term in the present symmetry consideration. According to the previous discussion, the current-induced modulation of a chemical-shift field (the IH effect) is ruled out by the symmetry restriction and the electric-field-induced modulation of that (the EH effect) is also ruled out by the result of the present field-reversal NMR measurements. Hence, we can neglect this field hereafter. The second, the trivial Oersted field, can occur in any materials under a macroscopic electric current. This field is not the hyperfine field of the current-induced orbital magnetization, because no orbital magnetization is accompanied with this field. Note that the trivial Oersted field does not alter the NMR spectra at all in an ideal case where a macroscopic electric current and a magnetic field is applied exactly parallel to the same direction, because the trivial Oersted field is perpendicular to the applied magnetic field in this case. Slight misalignments of the electric current and magnetic field from the $c$ axis in the present experiments would affect the NMR spectra, but they cause not shifts but symmetric line broadening of the spectra. Thus, the trivial Oersted field cannot explain the observed current-induced NMR shift (see Appendix D for more quantitative discussion about the trivial Oersted field). The third, the nontrivial magnetic field induced by the circular component of a microscopic solenoidal electric current should be interpreted as the hyperfine field of the current-induced orbital magnetization, which has been 
recently discussed theoretically [44,45]. In this case, a magnetic field due to the orbital magnetization is proportional to $\xi j_{z} S / c$, where $j_{z}$ is an electric current density, $c=5.93$ $\AA$ is the pitch of the tellurium helix, $S=4.50 \AA^{2}$ is the cross-sectional area of the helix, and $\xi$ is a dimensionless factor indicating an effective number of turns of a solenoidal electric current within a unit cell. The factor $\xi$ is unity when a system can be treated as classical solenoids and $\xi$ is enhanced with a factor of 10 at most in a tight-binding model calculation for a chiral system similar to tellurium [45]. Assuming $\xi=1-10$ and using the experimental values of $j_{z} \sim 100$ A $\mathrm{cm}^{-2}$, we obtain a very weak orbital hyperfine field of $10^{-6}-10^{-7} \mathrm{mT}$. Thus, we consider that this effect cannot be detected by the present experiment. Moreover, the polarity of the observed current-induced shift also indicates that the current-induced orbital magnetization does not dominate the observed shift. The orbital hyperfine coupling constant must be positive according to the conventional electromagnetics. In addition, theoretical studies [20,46] showed that the intrinsic orbital magnetization of each Bloch state of the highest valence bands is antiparallel to the spin magnetization of each state. This suggests that a current-induced orbital magnetization should cause a negative NMR shift for the left-handed crystal under a positive current, whereas an observed shift is positive. The insensitiveness of the NMR to the current-induced orbital magnetization allows us to separate the spin and orbital contributions to the currentinduced magnetization using the present NMR method and other probes that can detect the current-induced total magnetization.

\section{ACKNOWLEDGMENTS}

We thank Koji Tanaka for experimental assistance. This work was supported by JSPS KAKENHI Grants No. 17K14345, 18K03540, 19H02583, $19 \mathrm{H} 01852$ and a Grant for Basic Science Research Projects from the Sumitomo Foundation.

\section{APPENDIX A: METHOD}

\section{Sample Preparation}

Single crystals of trigonal tellurium were synthesized with the physical vapor transport (PVT) technique for sample 1 and the repeated Bridgman method for sample 2. For the PVT technique, elemental tellurium $(6 \mathrm{~N})$ grains of $0.46 \mathrm{~g}$ were sealed in an evacuated quartz tube heated in a two-zone tube furnace with temperatures set to $445^{\circ} \mathrm{C}$ (source side) and $395{ }^{\circ} \mathrm{C}$ (growth side). Single crystals that were typically $1 \mathrm{~mm}$ in size with well-defined faces were obtained after cooling, one of which was used for the NMR studies as sample 1. For the repeated Bridgman method, a single-crystal ingot was obtained after repeatedly melted at $600{ }^{\circ} \mathrm{C}$ in an evacuated quartz tube. After the crystal growth, the obtained ingot was crashed into pieces, one of which was used for the NMR studies as sample 2. The length along the $c$ axis of each sample $L$ and the cross-sectional area $S$ were approximately $L=\sim 2.6 \mathrm{~mm}$ and $S=\sim 0.33 \mathrm{~mm}^{2}(\sim 0.6 \mathrm{~mm} \times \sim$ $0.5 \mathrm{~mm}$ ) for sample 1 and $L=\sim 4.5 \mathrm{~mm}$ and $S=\sim 0.79 \mathrm{~mm}^{2}$ $(\sim 1.0 \mathrm{~mm} \times \sim 0.8 \mathrm{~mm})$ for sample 2 . The crystal structure chirality for each sample was investigated by observing the shapes of the etch pits [Figs. 3(a) and 5(a)] produced by the slow action of hot sulfuric acid $\left(100{ }^{\circ} \mathrm{C}, 30 \mathrm{~min}\right)$ on the cleavage planes of the crystals [29]. A pulsed electric current was applied approximately along the $c$ axis through two electrodes placed at the top and bottom of the sample. Titanium and gold were evaporated on the samples to achieve a good electrical contact between the sample and the electrodes. Copper wires were then attached using silver paste. For the NMR measurements, a coil was wound around each sample.

\section{NMR measurements}

The NMR spectra of ${ }^{125} \mathrm{Te}$ (nuclear spin $I=1 / 2$, gyromagnetic ratio $\gamma=13.454 \mathrm{MHz} / \mathrm{T}$ ) were measured at $100 \mathrm{~K}$ under an external magnetic field of $8.012 \mathrm{~T}$ for sample 1 and $8.327 \mathrm{~T}$ for sample 2. A magnetic field was applied almost parallel to the $c$ axis, but slightly tilted to the $y$ axis. The NMR spectra were obtained as a function of the resonance frequency $f$ by the Fourier transformation of the spin-echo signals following a $\pi / 2-\pi$ pulse sequence. All throughout this paper, the NMR spectra are shown as a function of the magnetic field felt by the ${ }^{125} \mathrm{Te}$ nuclei and calculated using $B(\mathrm{~T})=f(\mathrm{MHz}) / \gamma$. The NMR pulse sequence and the pulsed electric current were synchronized, such that the current pulse was switched on $50 \mu$ s of time before the NMR $\pi / 2$ pulse and switched off well after the decay of the spin-echo signals [16]. The polarities of an electric current and of a magnetic field are defined as positive when they direct a positive direction of a laboratory coordinate system. The first moment of the NMR spectrum $\delta$ was calculated from $\delta=\int I(B) B d B / \int I(B) d B$, whose interval of integration was the full width at half maximum of each line, where $I(B)$ is the spectral intensity at $B$.

\section{APPENDIX B: RELATION AMONG THE CRYSTAL STRUCTURE CHIRALITY AND THE PHYSICAL PROPERTIES OF TRIGONAL TELLURIUM}

In our previous study [16], we wrote that the highest valence band of the $P 3_{1} 21\left(D_{3}^{4}\right)$ right-handed tellurium has an inward spin-angular-momentum texture around the $H$ and $H^{\prime}$ points in the $\mathbf{k}$ space, while that of the $P 3_{2} 21\left(D_{3}^{6}\right)$ lefthanded tellurium has an outward texture. This relation was indirectly deduced from the relation between the $\mathbf{k}$ space spin texture and the natural optical rotatory power along the $c$ axis, which is the optic axis, and that between the natural optical rotatory power and the crystal structure chirality reported in past studies [Fig. 7(a)]. However, recent $a b$ initio calculations [20,21] and spin- and angle-resolved photoemission spectroscopy [21] independently showed that the opposite relation are probable, that is, the highest valence band of the $P 3_{1} 21\left(D_{3}^{4}\right)$ right-handed tellurium has the outward spin-angular-momentum texture in the $\mathbf{k}$ space, while that of the $P 3_{2} 21\left(D_{3}^{6}\right)$ left-handed tellurium has the inward spin-angular-momentum texture [Fig. 7(b)]. To understand the chirality-dependent physical properties of trigonal tellurium, this section briefly summarizes the relations among the crystal structure chirality and some physical properties of trigonal tellurium with reference to the previous studies. 
(a)

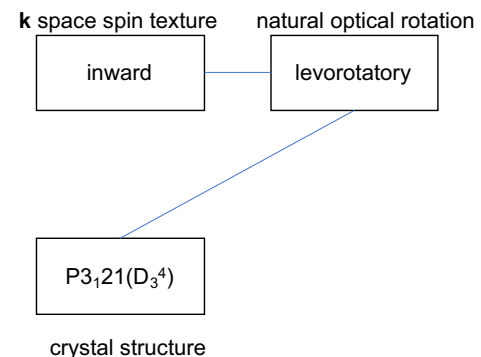

(b)

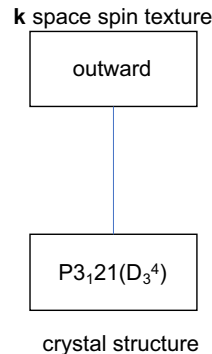

FIG. 7. Relations between the spin-angular-momentum texture and the crystal structure chirality. (a) Deduced from the past studies. (b) Relation claimed by the recent studies [20,21].

\section{Definitions and notations of the physical properties}

An unambiguous definition of the positive/negative signs (polarities) is crucially important in studying the physical properties and phenomena depending on the material chirality. Historically, confusion and contradiction caused by an ambiguous definition have been repeated in studies on chiral materials. Thus, we explicitly define the word herein and the sign of some physical properties.

Coordination. We use only the right-handed coordinates. Figure 1(a) depicts the relation among the $x, y$, and $z$ axes and the crystal axes of trigonal tellurium.

Crystal structure chirality. Trigonal tellurium belongs to the chiral space group of either $P 3_{1} 21\left(D_{3}^{4}\right)$ or $P 3_{2} 21\left(D_{3}^{6}\right)$. The screw operations $3_{1}$ and $3_{2}$ are called right- and lefthanded screws, respectively [47]. According to this notation, we call $P 3_{1} 21\left(D_{3}^{4}\right)$ tellurium as the "right-handed tellurium" and $P 3_{2} 21\left(D_{3}^{6}\right)$ tellurium as the "left-handed tellurium." (Note that one cannot straightforwardly define the chirality of an enantiomorphic crystal structure by referring to the symmetry elements when a given crystal structure belongs to one of the 43 achiral Sohncke groups.) We stress that, in principle, no $a$ priori relations exist among the chirality of a space group and that of the helices of dominant chemical bonds (e.g., $\alpha$ - and $\beta$-quartz [47]). However, for trigonal tellurium, the chiralities of the screw axes in the space group and of the helices of the dominant covalent bonds between the nearest neighbor atoms are the same, that is, $P 3_{1} 21\left(D_{3}^{4}\right)$ tellurium is composed of right-handed helices.

Angular-momentum texture in $\boldsymbol{k}$ space. The polarity of the angular-momentum texture in the $\mathbf{k}$ space and that of the magnetization texture must be clearly distinguished because the polarity of the angular momentum and that of magnetization are not always the same. For a spin part, the two quantities always have opposite signs for electrons. To avoid confusion, we discuss only the angular-momentum and the magnetization textures for electrons, not holes.

Notation for the optical properties. The four following properties have a notation related to the discussion about the absolute sign of the natural optical rotation: (1) Circularly polarized light. We use the term "right circularly polarized light" when the electric field vector rotates clockwise in time at a fixed place when viewed by an observer whose eye the light is entering [48]. (2) The phase of light: The notation of the phase of light affects the notation of the complex

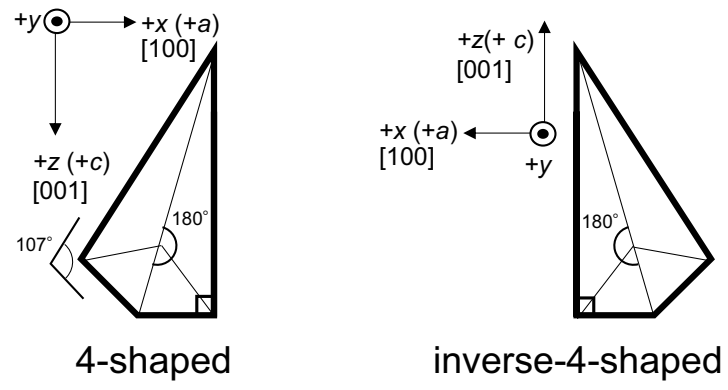

FIG. 8. Etch pit forms.

susceptibility and that of the polarization vectors (Jones vectors) of light. We use the notation of $\mathbf{k r}-\omega t$, not $\omega t-\mathbf{k r}$. That is, we represent complex dielectric permittivity as $\varepsilon=$ $\varepsilon_{1}+\mathrm{i} \varepsilon_{2}$ (not $\varepsilon_{1}-\mathrm{i} \varepsilon_{2}$ ) and the polarization vector of the right-handed polarized light as $(\hat{\mathbf{x}}-\mathrm{i} \hat{\mathbf{y}})$. (3) Optical rotation: We use the term dextrorotatory optical rotation when the polarization plane at an observer rotates clockwise from the polarization plane at a light source when viewed by an observer whose eye the light is entering [48]. (4) Sign of the optical rotatory power (sign of the rotatory angle): The optical rotatory power $\rho$ is defined as the rotatory angle $\theta$ divided by the crystal length $L$, in which the light propagates. We use the notation that $\rho, \theta>0$ when a levorotatory optical rotation (in our definition) is observed. Using the four notations, the optical rotatory power $\rho$ along the optic axis $z$ in the optical uniaxial crystal can be written as $\rho=\left(n_{\mathrm{R}}-n_{\mathrm{L}}\right) \omega / 2 c=$ $(\omega / 2 c) \operatorname{Im} \varepsilon_{x y}^{\mathrm{A}} / \sqrt{\varepsilon_{x x}}$, where $\omega$ is the angular frequency of light, $c$ is the speed of light in vacuum, $n_{\mathrm{R}}$ and $n_{\mathrm{L}}$ are the refractive indices for the right- and left-handed circularly polarized light, respectively, and $\varepsilon_{x x}$ and $\varepsilon_{x y}^{\mathrm{A}}$ are the $x x$ component and the antisymmetric part of the $x y$ (not $y x$ ) component of the dielectric tensor, respectively.

Etch pits. Trigonal tellurium has the characteristic enantiomorphic form of etch pits on the cleavage $\{01 \overline{1} 0\}$ prismatic faces. The etch pit chirality depends on that of the crystal structure. To avoid confusion, we do not use "left" and "right" to designate the etch pit form; instead, we use the terms "4shaped" and "inverse 4-shaped" to designate them as shown in Fig. 8 because the " 4 " resembles one of the etch pit forms.

\section{Summary of the previous studies}

The relations among the four properties, namely, crystal structure (space group), angular-momentum texture in $\mathbf{k}$ space, natural optical activity, and etch pit shape, should be determined to study the chirality-dependent physical properties of trigonal tellurium (Fig. 9). The latter two, natural optical activity and etch pits, are practically important because they are relatively easy to measure experimentally. Many studies determined the relation between two of the four properties. We summarized the previous studies below as far as we know to obtain the correct relations.

\section{a. Crystal structure and angular-momentum texture in $\boldsymbol{k}$ space}

Through ab initio calculations, Tsirkin et al. [20] and Sakano et al. [21] independently showed that the spin-angularmomentum texture of the highest valence band around the $H$ 


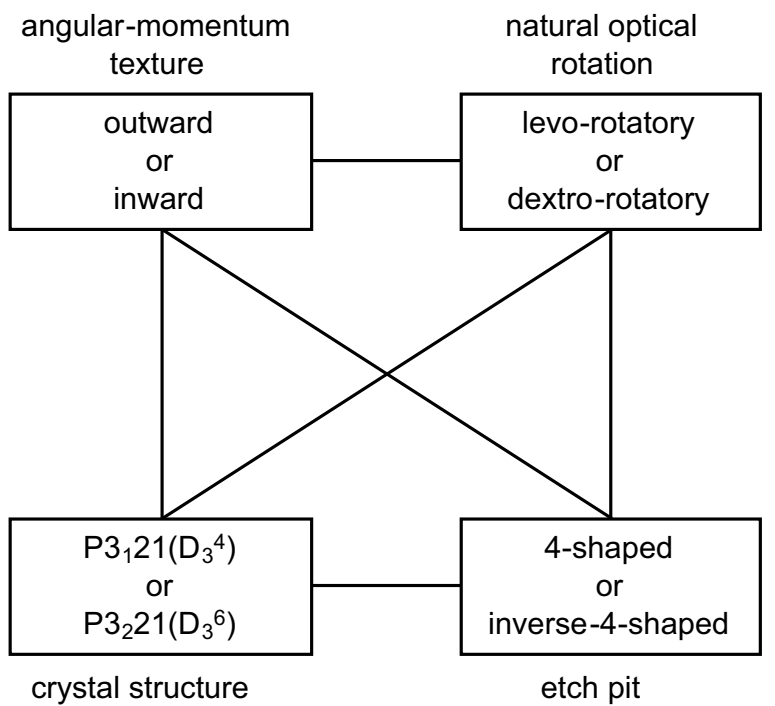

FIG. 9. Relations among the four properties, namely, crystal structure (space group), angular-momentum texture in $\mathbf{k}$ space, natural optical activity, and etch pit shape.

and $H^{\prime}$ points directs outward for $P 3_{1} 21\left(D_{3}^{4}\right)$ tellurium and inward for $P 3_{2} 21\left(D_{3}^{6}\right)$ tellurium. Theoretical studies [35,49] showed that the Bloch states of the highest valence band around the $H$ and $H^{\prime}$ points are primarily composed of states with the projected total angular momentum $j_{z}=+3 / 2$ and $-3 / 2$. Hence, the orbital-angular-momentum texture for the highest valence band around the $H$ and $H^{\prime}$ points are parallel to the spin-angular-momentum texture and should also direct outward for $P 3_{1} 21\left(D_{3}^{4}\right)$ tellurium and inward for $P 3_{2} 21\left(D_{3}^{6}\right)$ tellurium.

\section{b. Crystal structure and natural optical activity}

Two experimental studies and one theoretical study were performed for the relation between the crystal structure chirality and the natural optical activity chirality. For the experimental studies, Brown et al. first directly showed that a $P 3_{1} 21\left(D_{3}^{4}\right)$ crystal exhibits a levorotatory natural optical rotation [32]. In contrast, Tanaka et al. directly showed that a $P 3_{2} 21\left(D_{3}^{6}\right)$ crystal exhibits a levorotatory natural optical rotation [30,31]. Brown et al. measured the natural optical rotatory power and the Schwinger scattering intensities of polarized neutrons using an identical single crystal of trigonal tellurium. The latter neutron experiment provided information on the absolute crystal structure. Tanaka et al. measured the natural optical rotatory power and resonant diffraction with circularly polarized x-ray using an identical single crystal. The latter's x-ray experiment also provided information on the absolute crystal structure. The measurement temperatures and the carrier density of the samples for the natural optical rotatory power measurements were not explicitly written in both reports. However, the degrees of the natural optical rotatory power observed in both studies reproduced the experimental results for nondoped samples at room temperature [24,25]. This indicates that sign change of the natural optical rotatory power depending on the measurement temperatures and the carrier densities is very unlikely between the two reports; thus, the two results are totally contradicting, and one of the two claims must be modified. As for the theoretical study, Reijnhart claimed that a $P 3_{1} 21\left(D_{3}^{4}\right)$ crystal shows a dextrorotatory natural optical rotatory power [50]. He calculated the natural optical rotatory power of trigonal tellurium and other materials having the helical structures similar to trigonal tellurium and belonging to $P 3_{1} 21\left(D_{3}^{4}\right)$ space group (e.g., $\alpha$-quartz) by using the classical point dipole approximation. The theoretical results for the $\alpha$-quartz correctly reproduced the chirality of their natural optical rotatory power that was measured experimentally. The theoretical result that $P 3_{1} 21\left(D_{3}^{4}\right)$ tellurium has a dextrorotatory natural optical rotatory power is consistent with the resonant $\mathrm{X}$-ray study by Tanaka et al. and inconsistent with the neutron study by Brown et al.

\section{c. Crystal structure and etch pits}

Koma et al. claimed that $P 3_{1} 21\left(D_{3}^{4}\right)$ crystals show 4shaped etch pits, while $P 3_{2} 21\left(D_{3}^{6}\right)$ crystal shows inverse 4-shaped etch pits [29]. They observed the chiral shaped etch pits on the cleavage $\{01 \overline{1} 0\}$ prismatic faces of single crystals, which were etched by a slow attack of concentrated sulfuric acid. Considering a microscopic atomic arrangement, they deduced that the etch pits on the cleavage $\{01 \overline{1} 0\}$ prismatic faces of $P 3_{1} 21\left(D_{3}^{4}\right)$ tellurium can form a 4-shaped type with a specific internal form, which was called Model I in their report. They also deduced that the etch pits for $P 3_{2} 21\left(D_{3}^{6}\right)$ tellurium can form mirror images of the etch pits for $P 3_{1} 21\left(D_{3}^{4}\right)$ tellurium. They experimentally observed that the etch pits for a given single crystal have the form of either a 4-shaped or an inverse 4-shaped type, which had the specific internal form of Model I (Fig. 8). Thus, they concluded that $P 3_{1} 21\left(D_{3}^{4}\right)$ tellurium shows 4-shaped etch pits, and $P 3_{2} 21\left(D_{3}^{6}\right)$ tellurium shows inverse 4-shaped etch pits. According to the consideration of a microscopic atomic arrangement, they also claimed that an etch pit form indicates the positive direction of the $x$ axis ([100] direction) (Fig. 8). They measured the angle dependence of the chemical shifts of the ${ }^{125} \mathrm{Te}$ NMR lines for single crystals with different etch pit forms [29,33]. They confirmed that the relation between the angle dependence of the chemical shifts, which depends on the crystal chirality, and the positive $x$ direction predicted by the etch pit forms is consistent with the relation calculated by Shimizu [51], which was based on a tight binding approximation for the $5 p$ orbitals of tellurium. This consistency also supports their claim for the relation between the crystal structure chirality and the etch pit form.

\section{d. Angular-momentum texture in $k$ space and etch pits}

Sakano et al. performed spin- and angle-resolved photoelectron spectroscopy measurement for two crystals; one of which has 4-shaped etch pits, while the other has inverse 4-shaped etch pits [21]. The etch pits were formed by hot concentrated sulfuric acid. They directly determined the spinmagnetization texture of the highest valence band for the two crystals and observed that the crystal with 4-shaped etch pits had an inward spin-magnetization texture, namely the outward spin-angular-momentum texture, and that the crystal with an inverse 4-shaped etch pit has the inward spin-angularmomentum texture. 


\section{e. Natural optical activity and etch pits}

Three different groups reported that crystals with 4-shaped etch pits have a levorotatory natural optical rotatory power. Blakemore and Nomura first claimed that a crystal with 4shaped etch pits produced by the slow action of hot sulfuric acid has a levorotatory optical rotatory power [52]. (Note that we could not find the definition of the optical rotatory power in their paper; thus, ambiguity in the definition remains.) The measurement temperature and the carrier density for the crystals used for the optical activity measurement were not reported in the paper. Fukuda et al. [23] and Ades et al. [24] also claimed that crystals with 4-shaped etch pits produced by the slow action of hot sulfuric acid show a levorotatory natural optical rotatory power. (Note that the two groups most likely used the definition of chirality of the optical rotatory power according to our definition.) The measurement temperatures and the carrier densities of crystals in the study by Fukuda et al. were not explicitly described. The measurement by Ades et al. was done for a nondoped crystal at room temperature.

\section{f. Angular-momentum texture in $k$ space and natural optical activity}

Previous studies claimed that the outward angularmomentum texture of the highest valence band causes a dextrorotatory natural optical rotatory. Ivchenko and Pikus first showed the relation by calculating the natural optical rotatory power caused by the interband transition between the highest valence band and the conduction bands [49]. (In the following section, the $z$ axis component of the propagation vector of the light $q_{\mathrm{z}}$ is fixed to a positive value.) They showed that the $x y z$ component of the third-rank gyration tensor, $\gamma_{x y z}=\operatorname{Im} \varepsilon_{x y}^{\mathrm{A}} / q_{z}$, has the opposite sign of the coefficient of the linear dispersion term of the highest valence band, $S$ [Eq. (15) in [49]]. The linear dispersion term $S k_{z}$ appears in the effective energy dispersion of the highest valence band near the $H$ and $H^{\prime}$ points, $E(\mathbf{k})=\lambda_{\perp}\left(k_{x}^{2}+k_{y}^{2}\right)+\lambda_{\|} k_{z}^{2}+\sqrt{S^{2} k_{z}^{2}+\Delta^{2}}$, where $\mathbf{k}=\left(k_{x}, k_{y}, k_{z}\right)$ is a wave number measured from the $H$ or $H^{\prime}$ points. The eigenstates for the highest valence band at $\mathbf{k}$, where $\left|k_{x}\right|,\left|k_{y}\right| \ll\left|k_{z}\right|$ near the $H$ and $H^{\prime}$ points, are represented as $1 / \sqrt{2} \times\left(1+S k_{z} / \sqrt{S^{2} k_{z}^{2}+\Delta^{2}}\right)^{1 / 2} \mid j_{z}=$ $+3 / 2>+1 / \sqrt{2} \times\left(1-S k_{z} / \sqrt{S^{2} k_{z}^{2}+\Delta^{2}}\right)^{1 / 2} \mid j_{z}=-3 / 2>$, where $\mid j_{z}=+3 / 2>$ and $\mid j_{z}=-3 / 2>$ are the states with the $c$-axis total projected angular momentum $+3 / 2$ and $-3 / 2$, respectively. Thus, the total-angular-momentum texture of the highest valence band directs outward from the $H$ and $H^{\prime}$ points when $S>0$ (inward when $S<0$ ). Similar to the angular-momentum texture, the sign of $S$ also depends on the crystal structure chirality. A dextrorotatory natural optical rotatory occurs when $\gamma_{x y z}<0$; thus, their calculations indicate that the outward spin texture causes a dextrorotatory natural optical rotatory.

Shalygin et al. measured the optical rotatory power of a single crystal under an electric current at $T=77 \mathrm{~K}$ and $\hbar \omega=0.117$ to $0.238 \mathrm{eV}$ [15], in which the hole density of the sample was $4 \times 10^{16} \mathrm{~cm}^{-3}$. They demonstrated that the natural optical rotatory angle for the positive propagation vector $\mathbf{q} \|+\hat{\mathbf{z}}$ and the current-induced optical rotatory angle under an electric current that directs parallel (not antiparallel) to the propagation vector $(\mathbf{I} \| \mathbf{q})$ have opposite signs. Note that this relation is not altered by the ambiguity of the notations of the optical properties. They also calculated the current-induced optical rotatory power of nondegenerate tellurium under an electric current $(\mathbf{I}\|\mathbf{q}\|+\hat{\mathbf{z}})$ based on the semiclassical theory using the effective band dispersions with the coefficient of the linear dispersion term $S$, neglecting the natural optical rotatory power. They obtained that the current-induced levorotatory optical activity $\left(\operatorname{Im} \varepsilon_{x y}^{\mathrm{A}}>0\right.$ ) occurs for $S>0$ when an electric current is positive. Combining their experimental and theoretical results, the outward angular-momentum texture of the highest valence band causes a dextrorotatory natural optical activity.

Most recently, Tsirkin [20] also calculated the natural optical activity caused by inter- and intra-band transitions through $a b$ initio calculations. They showed that $P 3_{1} 21\left(D_{3}^{4}\right)$ tellurium, which has an outward angular-momentum texture, exhibited a dextrorotatory natural optical rotation primarily caused by the interband transition at $T=77 \mathrm{~K}$ and $\hbar \omega=0.117 \mathrm{eV}$ when the hole density is low $\left(n<10^{18} \mathrm{~cm}^{-3}\right)$. They also found that a levorotatory natural optical rotation appears at the limited range of the carrier density $\left(10^{18} \mathrm{~cm}^{-3}<n<10^{19} \mathrm{~cm}^{-3}\right)$, although the sign change of the natural optical activity by doping or temperature change has not been experimentally found so far [25]. They also calculated the current-induced optical rotatory power and found that it has the opposite sign to the natural optical rotatory power when light travels parallel to the current $(\mathbf{I}\|\mathbf{q}\|+\hat{\mathbf{z}})$, which agrees with the experimental results by Shalygin et al. [15]. The calculated $|\rho|$ for the natural and the current-induced optical rotatory power are smaller by approximately a factor of two compared to the experimentally measured values.

\section{Possible relations in the contradiction between previous reports}

Figure 10 shows all possible relations among the crystal structure chirality, $\mathbf{k}$ space angular-momentum texture, natural optical rotatory, and etch pit form. We compared each relation with the previous studies in Fig. 10 to evaluate which relation is the most probable. Relations A and B are the most probable because they have the fewest number of reports that should be ruled out or modified. Importantly, for both cases, the relations among the structure chirality, angularmomentum texture, and etch pit form are the same. Thus, both relations $\mathrm{A}$ and $\mathrm{B}$ are consistent with our present conclusion in the main text, that is, when an electric current was applied to the positive direction, a positive NMR shift or a positive spin magnetization was found in $P 3_{2} 21\left(D_{3}^{6}\right)$ left-handed tellurium with an inverse-4-shaped etch pit, and a negative NMR shift was found in $P 3_{1} 21\left(D_{3}^{4}\right)$ right-handed tellurium with a 4-shaped etch pit.

\section{APPENDIX C: POSSIBILITY OF THE MULTIDOMAIN NATURE OF THE INGOT OF TELLURIUM}

In our previous study [16], we reported that we used a single crystal of $P 3_{1} 21\left(D_{3}^{4}\right)$ right-handed tellurium for the NMR measurement under an electric current. However, the crystal used was $P 3_{2} 21\left(D_{3}^{6}\right)$ left-handed tellurium. We prepared the single crystal by crashing a tellurium ingot grown by the 

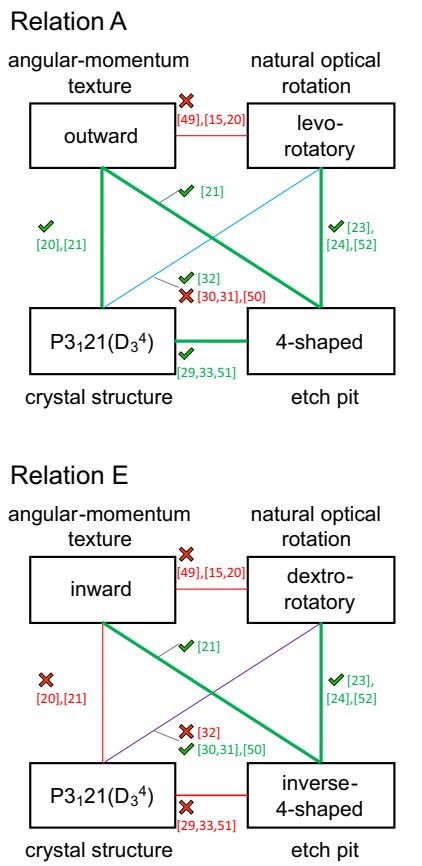
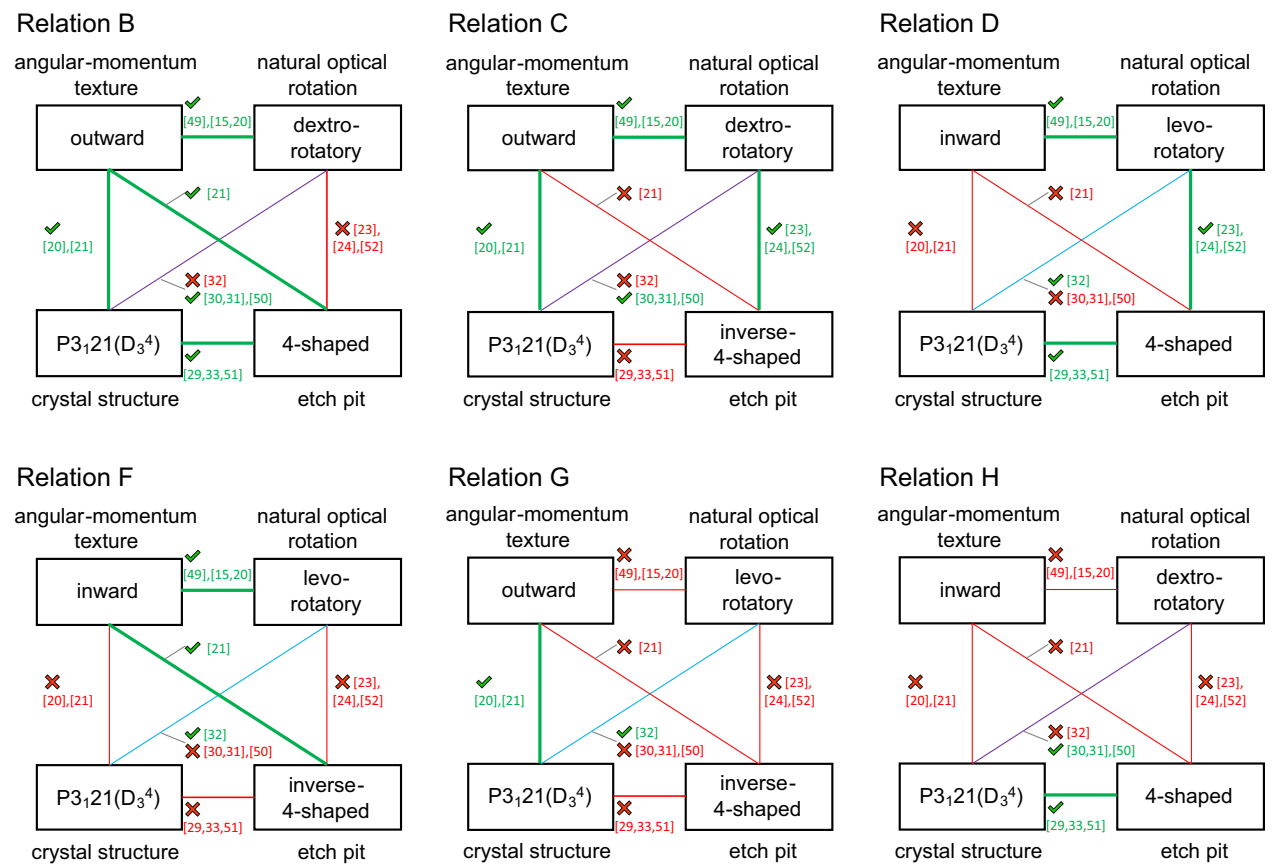

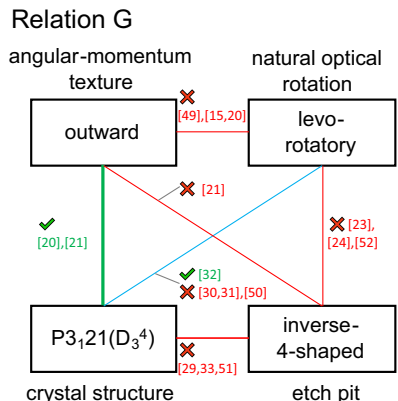

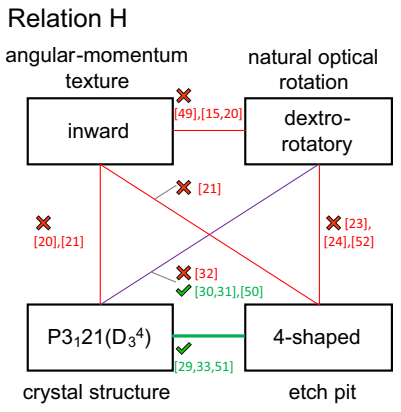

FIG. 10. All possible relations among the four physical properties. The chirality of the crystal structure $P 3_{1} 21\left(D_{3}^{4}\right)$ is fixed for brevity. If relations for $P 3_{2} 21\left(D_{3}^{6}\right)$ are needed, all other properties should be inverted (e.g., levorotatory $\leftrightarrow$ dextrorotatory, inward texture $\leftrightarrow$ outward texture, and 4-type $\leftrightarrow$ inverse 4-type). The relation connected by a green (red) line is (not) consistent with all the related previous studies. The relation connected by a blue or a purple line is consistent with a different part of the previous studies.

Bridgman method without seed crystal. We observed 4-shaped etch pits on the surfaces of the other pieces of the ingot; thus, we thought that all pieces of the ingot had a right-handed chirality of the crystal structure and that the single crystal used was accordingly a right-handed crystal $\left[P 3_{1} 21\left(D_{3}^{4}\right)\right]$. However, we recently observed that some pieces of the ingot had inverse 4-shaped etch pits; thus, we concluded anew that the ingot has multichirality domains. Unfortunately, the etch pits of the crystal used in the previous study cannot be measured because the crystal was destroyed during attempts to remove it from the epoxy in which the crystal was embedded. The previous study showed that the current-induced NMR shift of the crystal was positive under a positive electric current. Comparing this result with the present finding that a positive electric current causes a positive current-induced NMR shift for $P 3_{2} 21\left(D_{3}^{6}\right)$ tellurium and a negative shift for $P 3_{1} 21\left(D_{3}^{4}\right)$ tellurium, we eventually conclude that the single crystal used in the previous study was a $P 3_{2} 21\left(D_{3}^{6}\right)$ left-handed crystal.

\section{APPENDIX D: ESTIMATION OF THE TRIVIAL OERSTED FIELD INDUCED BY A MACROSCOPIC ELECTRIC CURRENT}

In the main text, we conclude that the origin of currentinduced NMR shift is not the Oersted field but the hyperfine field due to the current-induced magnetization. This is because the Oersted field cause the spectral broadening not a shift, assuming an ideal condition. Here we discuss the effect of the Oersted field more correctly. First of all, we estimate the trivial Oersted field $\mathbf{H}_{\text {Oersted }}$ when an applied electric current density $j$ is exactly parallel to an external magnetic field $\mathbf{H}_{0}=\left(0,0, H_{0}\right)$. We assume that a sample shape is an ideal thin rod for simplicity. The estimated field at $\mathbf{r}=(x, y, z)$ is $\mathbf{H}_{\text {Oersted }}(\mathbf{r})=j / 2 \times(-y, x, 0)$. Thus, the trivial Oersted field does not alter the NMR spectra at all in this ideal case because of the absence of the $z$-axis component of the trivial Oersted field. In the present experiment, an external magnetic field was slightly tilted to the $y$ axis; thus an electric current and a magnetic field is not exactly parallel to each other. Accordingly, the misalignment angle $\Delta \theta$ (defined in the $x z$ plane) can cause a finite $z$-axis component of the trivial Oersted magnetic field $H_{\text {Oersted, } z}(\mathbf{r})=j / 2 \times x \times \sin \Delta \theta$. Using the experimental value of sample $1, \Delta \theta=7^{\circ}$ and $j=182 \mathrm{~A} \mathrm{~cm}^{-2}, H_{\text {Oersted }, z}(\mathbf{r})$ is estimated to $0.14 \times x(\mathrm{~mm}) \mathrm{mT}$. Thus, the maximum increase in the square root of the second moment of the NMR spectra due to the $z$-axis component of the trivial Oersted field is estimated to $\Delta H_{\text {Oersted, } z}^{\max }=0.02 \mathrm{mT}$, when we use the radius of sample 1 . This value is less than the induced NMR shift when $j=182 \mathrm{~A} \mathrm{~cm}^{-2}$. More importantly, the trivial Oersted field in this case causes only symmetric broadening of the NMR spectra within $\pm 0.02 \mathrm{mT}$, not a shift. Even though there is something causing asymmetry of the trivial Oersted field, for example, asymmetry of the sample shape or electric-current distribution, we at least conclude that the shift (not the broadening) caused by the trivial Oersted field is much less than $0.02 \mathrm{mT}$; thus, the current-induced NMR shift cannot be explained by the trivial Oersted field quantitatively. Using the parameter of sample 2 also yields the same conclusion. 
[1] I. E. Dzyaloshinskii, On the magneto-electrical effect in antiferromagnets, J. Exp. Theor. Phys. 37, 881 (1959).

[2] More precisely, in addition to inversion and time-reversal symmetry, some combinations of other crystal symmetries(proper and improper) rotation, translation, and nonsymmorphic symmetry - and their anti-unitary counterparts can also forbid the linear electric-field-induced magnetization. Consequently, only 58 of 122 magnetic point groups allow the linear electric-field-induced magnetization effect [53].

[3] S. D. Ganichev, E. L. Ivchenko, V. V. Bel'kov, S. A. Tarasenko, M. Sollinger, D. Weiss, W. Wegscheider, and W. Prettl, Spingalvanic effect, Nature (London) 417, 153 (2002).

[4] Y. K. Kato, R. C. Myers, A. C. Gossard, and D. D. Awschalom, Current-Induced Spin Polarization in Strained Semiconductors, Phys. Rev. Lett. 93, 176601 (2004).

[5] V. Sih, R. C. Myers, Y. K. Kato, W. H. Lau, A. C. Gossard, and D. D. Awschalom, Spatial imaging of the spin Hall effect and current-induced polarization in two-dimensional electron gases, Nat. Phys. 1, 31 (2005).

[6] A. Y. Silov, P. A. Blajnov, J. H. Wolter, R. Hey, K. H. Ploog, and N. S. Averkiev, Current-induced spin polarization at a single heterojunction, Appl. Phys. Lett. 85, 5929 (2004).

[7] C. L. Yang, H. T. He, L. Ding, L. J. Cui, Y. P. Zeng, J. N. Wang, and W. K. Ge, Spectral Dependence of Spin Photocurrent and Current-Induced Spin Polarization in an InGaAs/InAlAs TwoDimensional Electron Gas, Phys. Rev. Lett. 96, 186605 (2006).

[8] N. P. Stern, S. Ghosh, G. Xiang, M. Zhu, N. Samarth, and D. D. Awschalom, Current-Induced Polarization and the Spin Hall Effect at Room Temperature, Phys. Rev. Lett. 97, 126603 (2006).

[9] Z. Wilamowski, H. Malissa, F. Schäffler, and W. Jantsch, $g$-Factor Tuning and Manipulation of Spins by an Electric Current, Phys. Rev. Lett. 98, 187203 (2007).

[10] W. F. Koehl, M. H. Wong, C. Poblenz, B. Swenson, U. K. Mishra, J. S. Speck, and D. D. Awschalom, Current-induced spin polarization in gallium nitride, Appl. Phys. Lett. 95, 072110 (2009).

[11] H. J. Zhang, S. Yamamoto, Y. Fukaya, M. Maekawa, H. Li, A. Kawasuso, T. Seki, E. Saitoh, and K. Takanashi, Current-induced spin polarization on metal surfaces probed by spin-polarized positron beam, Sci. Rep. 4, 4844 (2014).

[12] S. D. Ganichev and L. E. Golub, Interplay of Rashba /Dresselhaus spin splittings probed by photogalvanic spectroscopy-A review, Phys. Status Solidi (b) 251, 1801 (2014).

[13] V. M. Edelstein, Spin polarization of conduction electrons induced by electric current in two-dimensional asymmetric electron systems, Solid State Commun. 73, 233 (1990).

[14] L. E. Vorobev, E. L. Ivchenko, G. E. Pikus, I. I. Farbshtein, V. A. Shalygin, and A. V. Shturbin, Optical activity in tellurium induced by a current, Pis'ma Zh. Eksp. Teor. Fiz. 29, 485 (1979) [JETP Lett. 29, 441 (1979)].

[15] V. A. Shalygin, A. N. Sofronov, L. E. Vorob'ev, and I. I. Farbshtein, Current-induced spin polarization of holes in tellurium, Phys. Solid State 54, 2362 (2012).

[16] T. Furukawa, Y. Shimokawa, K. Kobayashi, and T. Itou, Observation of current-induced bulk magnetization in elemental tellurium, Nat. Commun. 8, 954 (2017).

[17] R. Naaman and D. H. Waldeck, Chiral-induced spin selectivity effect, J. Phys. Chem. Lett. 3, 2178 (2012).
[18] In principle, the chirality of the helices of dominant chemical bonds and that of the space-group screw axes are not always the same (e.g., $\beta$-quartz).

[19] M. Hirayama, R. Okugawa, S. Ishibashi, S. Murakami, and T. Miyake, Weyl Node and Spin Texture in Trigonal Tellurium and Selenium, Phys. Rev. Lett. 114, 206401 (2015).

[20] S. S. Tsirkin, P. A. Puente, and I. Souza, Gyrotropic effects in trigonal tellurium studied from first principles, Phys. Rev. B 97, 035158 (2018).

[21] M. Sakano, M. Hirayama, T. Takahashi, S. Akebi, M Nakayama, K. Kuroda, K. Taguchi, T. Yoshikawa, K. Miyamoto, T. Okuda, K. Ono, H. Kumigashira, T. Ideue, Y. Iwasa, N. Mitsuishi, K. Ishizaka, S. Shin, T. Miyake, S. Murakami, T. Sasagawa, and T. Kondo, Radial Spin Texture in Elemental Tellurium with Chiral Crystal Structure, Phys. Rev. Lett. 124, 136404 (2020).

[22] K. C. Nomura, Optical Activity in Tellurium, Phys. Rev. Lett. 5, 500 (1960).

[23] S. Fukuda, T. Shiosaki, and A. Kawabata, Infrared optical activity in tellurium, Phys. Status Solidi (b) 68, K107 (1975).

[24] S. Ades and C. H. Champness, Optical activity of tellurium to $20 \mu \mathrm{m}$, J. Opt. Soc. Am. 65, 217 (1975).

[25] H. Stolze, M. Lutz, and P. Grosse, The optical activity of tellurium, Phys. Status Solidi (b) 82, 457 (1977).

[26] M. Cheng, S. Wu, Z.-Z. Zhu, and G.-Y. Guo, Large secondharmonic generation and linear electro-optic effect in trigonal selenium and tellurium, Phys. Rev. B 100, 035202 (2019).

[27] G. Arlt and P. Quadflieg, Electronic displacement in tellurium by mechanical strain, Phys. Status Solidi (b) 32, 687 (1969).

[28] D. Royer and E. Dieulesaint, Elastic and piezoelectric constants of trigonal selenium and tellurium crystals, J. Appl. Phys. 50, 4042 (1979).

[29] A. Koma, E. Takimoto, and S. Tanaka, Etch pits and crystal structure of tellurium, Phys. Stat. Sol. 40, 239 (1970).

[30] Y. Tanaka, S. P. Collins, S. W. Lovesey, M. Matsumami, T. Moriwaki, and S. Shin, Determination of the absolute chirality of tellurium using resonant diffraction with circularly polarized x-rays, J. Phys.: Condens. Matter 22, 122201 (2010).

[31] Y. Tanaka, S. P. Collins, S. W. Lovesey, M. Matsunami, T. Moriwaki, and S. Shin, Determination of the absolute chirality of tellurium using resonant diffraction with circularly polarized X-rays, J. Phys.: Condens. Matter 24, 159501 (2012).

[32] P. J. Brown and J. B. Forsyth, The crystal structure and optical activity of tellurium, Acta Crystallogr. A: Found. Crystallogr. 52, 408 (1996).

[33] A. Koma, Anisotropic chemical shift of nuclear magnetic resonance in single crystals of tellurium and selenium, Phys. Status Solidi (b) 56, 655 (1973).

[34] O. Betbeder-Matibet and M. Hulin, A semi-empirical model for the valence band structure of tellurium, Phys. Stat. Sol. 36, 573 (1969).

[35] T. Doi, K. Nakao, and H. Kamimura, The valence band structure of tellurium. I. The $k \cdot p$ perturbation method, J. Phys. Soc. Jpn. 28, 36 (1970).

[36] Even in a crystal that lacks full rotational symmetry but has discrete rotational or screw symmetries, the (crystal) orbital angular momentum projected to the rotational or screw symmetry axis can be defined as $l_{z}=m \bmod n$ for a spinless system, where integer $m$ appears in the phase of $\pm 2 m \pi$ acquired by a wave function through the $n$-time operation of the $n$-fold rotation or 
the $n$-fold screw operations equivalent to the identity operation, where + and - are for the left- and right-handed symmetry operations, respectively. For $P 3_{1} 21\left(D_{3}^{4}\right)$ right-handed tellurium without the spin-orbit interaction, the degenerate states and the nondegenerate state at the $H\left(H^{\prime}\right)$ point, respectively, acquire the phases of $\pm 2 \pi$ and 0 , through the triple operation of the threefold left-handed screw operation $\left\{\rho_{3}^{-1} \mid 2 \mathbf{c} / 3\right\}$. [Note that the triple operation of the threefold right-handed screw operation $\left\{\rho_{3} \mid \mathbf{c} / 3\right\}$, which is another symmetry operation of $P 3_{1} 21\left(D_{3}^{4}\right)$, is not equivalent to the identity operation for the Bloch states at the $H\left(H^{\prime}\right)$ point because it provides the phases of $+3 \pi$ and $-\pi$ for the degenerate bands and $+\pi$ for the nondegenerate band.] Hence, the degenerate states and the nondegenerate states at the $H\left(H^{\prime}\right)$ point have $l_{z}= \pm 1$ and $l_{z}=0$, respectively [Fig. 2(c)].

[37] K. Nakayama, M. Kuno, K. Yamauchi, S. Souma, K. Sugawara, T. Oguchi, T. Sato, and T. Takahashi, Band splitting and Weyl nodes in trigonal tellurium studied by angle-resolved photoemission spectroscopy and density functional theory, Phys. Rev. B 95, 125204 (2017).

[38] T. Ideue, M. Hirayama, H. Taiko, T. Takahashi, M. Murase, T. Miyake, S. Murakami, T. Sasagawa, and Y. Iwasa, Pressureinduced topological phase transition in noncentrosymmetric elemental tellurium, Proc. Nat. Acad. Sci. USA 116, 25530 (2019).

[39] Note that conversion between $\mathbf{E}$ and $\mathbf{I}$ through Ohm's law leads to wrong conclusion on the viewpoint of symmetry. This is because a response tensor of a dissipative phenomenon (e.g., the symmetric part of a conductivity tensor) connects the $T$-even and $T$-odd quantities (e.g., $\mathbf{E}$ and $\mathbf{I}$, respectively) regardless whether a system has the timer-reversal symmetry or not.

[40] H. Selbach, O. Kanert, and D. Wolf, NMR investigation of the diffusion and conduction properties of the semiconductor tellurium. I. Electronic properties, Phys. Rev. B 19, 4435 (1979).

[41] K. von Klitzing and G. Landwehr, Surface quantum states in tellurium, Solid State Commun. 9, 2201 (1971).
[42] N. S. Averkiev, V. A. Berezovets, G. E. Pikus, N. I. Sablina, and I. I. Farbshtein, Quantum corrections to 2D hole conductivity in a quantum well on (1010), Phys. Solid State 40, 1409 (1998).

[43] K. Akiba, K. Kobayashi, T. C. Kobayashi, R. Koezuka, A. Miyake, J. Gouchi, Y. Uwatoko, and M. Tokunaga, Magnetotransport properties of tellurium under extreme conditions, Phys. Rev. B 101, 245111 (2020).

[44] T. Yoda, T. Yokoyama, and S. Murakami, Current-induced orbital and spin magnetizations in crystals with helical structure, Sci. Rep. 5, 12024 (2015).

[45] T. Yoda, T. Yokoyama, and S. Murakami, Orbital Edelstein effect as a condensed-matter analog of solenoids, Nano Lett. 18, 916 (2018).

[46] C. Şahin, J. Rou, J. Ma, and D. A. Pesin, Pancharatnam-Berry phase and kinetic magnetoelectric effect in trigonal tellurium, Phys. Rev. B 97, 205206 (2018).

[47] A. M. Glazer and K. Stadnicka, On the origin of optical activity in crystal structures, J. Appl. Crystallogr. 19, 108 (1986).

[48] J. F. Nye, Physical Properties of Crystals (Clarendon Press, Oxford, 1985).

[49] E. L. Ivchenko and G. E. Pikus, Natural optical activity of semiconductors (tellurium), Fiz. Tverd. Tela (Leningrad) 16, 1933 (1974) [Sov. Phys. Solid State 16, 1261 (1975)].

[50] R. Reijnhart, Classical calculations concerning the double refractions, optical rotation and absolute configuration, Ph.D. thesis, Delft University, 1970.

[51] T. Shimizu, A note on the anisotropic chemical shift of tellurium, Memoirs of the Faculty of Technology, Kanazawa University (Japan), 5, 189 (1969).

[52] J. S. Blakemore and K. C. Nomura, Enantiomorphous character of etch pits in tellurium, J. Appl. Phys. 32, 745 (1961).

[53] J.-P. Rivera, A short review of the magnetoelectric effect and related experimental techniques on single phase (multi-) ferroics, Eur. Phys. J. B 71, 299 (2009). 